\title{
Mean-field and Monte Carlo calculations of the three-dimensional structure factor for YBa2Cu3O6+X
}

Fiig, Thomas; Andersen, Niels Hessel; Lindgård, Per-Anker; Berlin, J.; Mouritsen, Ole G.

Published in:

Physical Review B Condensed Matter

Link to article, DOI:

10.1103/PhysRevB.54.556

Publication date:

1996

Document Version

Publisher's PDF, also known as Version of record

Link back to DTU Orbit

Citation (APA):

Fiig, T., Andersen, N. H., Lindgård, P-A., Berlin, J., \& Mouritsen, O. G. (1996). Mean-field and Monte Carlo calculations of the three-dimensional structure factor for $\mathrm{YBa}_{2} \mathrm{Cu}_{3} \mathrm{O}_{6+\mathrm{x}}$. Physical Review $B$ Condensed Matter,

\section{General rights}

Copyright and moral rights for the publications made accessible in the public portal are retained by the authors and/or other copyright owners and it is a condition of accessing publications that users recognise and abide by the legal requirements associated with these rights.

- Users may download and print one copy of any publication from the public portal for the purpose of private study or research.

- You may not further distribute the material or use it for any profit-making activity or commercial gain

- You may freely distribute the URL identifying the publication in the public portal 


\title{
Mean-field and Monte Carlo calculations of the three-dimensional structure factor for $\mathrm{YBa}_{2} \mathrm{Cu}_{3} \mathrm{O}_{6+x}$
}

\author{
T. Fiig, ${ }^{*}$ N. H. Andersen, and P.-A. Lindgård \\ Department of Solid State Physics, Risф National Laboratory, DK-4000 Roskilde, Denmark \\ J. Berlin \\ Thinking Machines Corporation, 245 First Street, Cambridge, Massachusetts 02142-1264 \\ O. G. Mouritsen \\ Department of Physical Chemistry, The Technical University of Denmark, DK-2800 Lyngby, Denmark
}

(Received 23 January 1996)

\begin{abstract}
We develop a general mean-field matrix theory for calculating phase boundaries and structure factors for a class of lattice gas Hamiltonians. We investigate the oxygen ordering in $\mathrm{YBa}_{2} \mathrm{Cu}_{3} \mathrm{O}_{6+x}$ by applying the theory and by Monte Carlo simulation using an extension to three dimensions of the two-dimensional anisotropic next nearest neighbor lattice gas model. The calculation of the structure factor in three spatial dimensions on a $256 \times 256 \times 16$ system has been implemented on a massively parallel computer, the Connection Machine CM2. This allows for an extremely accurate determination of the line shape of the structure factor in the entire reciprocal space in excellent agreement with the mean-field prediction. We report on the results for an oxygen stoichiometry of $x=0.4, x=0.5$, and $x=0.6$ for temperatures between $T=450$ and $800 \mathrm{~K}$. Using a sum-rule argument we have calculated the fluctuation-renormalized transition temperature into the ortho-II phase, $T_{c}$, in good agreement with Monte Carlo simulation results. This allows an analytic description of the dependence of $T_{c}$ on the interaction $V_{4}$ in the third dimension and on the oxygen stoichiometry $x$. An explanation for the experimental observation of the lack of a Bragg peak in the ordered ortho-II phase of $\mathrm{YBa}_{2} \mathrm{Cu}_{3} \mathrm{O}_{6+x}$ is offered in terms of scattering from platelike antiphase oxygen-ordered domains. [S0163-1829(96)06222-4]
\end{abstract}

\section{INTRODUCTION}

The physical properties of complex systems, e.g., with disordered surface and bulk structures, artificial multilayers, structure on a mesoscopic scale, and nonequilibrium phenomena, have been the focus of solid state physics in recent years. Experimental studies of the static and kinetic ordering properties of such systems have become possible via the rapid development of highly advanced structural techniques which supply increasingly higher resolution and shorter sampling times. The techniques include real space methods like scanning tunnel microscopy (STM) atomic force microscopy (AFM) and high-resolution transmission electron microscopy (TEM), and indirect ( $q$-space) diffraction techniques, where new powerful neutron, electron beam, and in particular x-ray synchrotron sources have opened for innovative studies. The neutron and x-ray diffraction techniques have significant advantages for nondestructive studies, and they may supply information on the bulk of these materials.

Interesting structural information is often contained in the details of the diffuse scattering, including also the small angle scattering, which is directly proportional to the diffuse part of the structure factor, $S_{\text {dif }}(\mathbf{q})$. However, $S_{\text {dif }}(\mathbf{q})$ is difficult to interpret and convert into the corresponding behavior in real space. Computer simulations may be used as an important tool to bridge this gap in our understanding. With the increasingly more powerful computers becoming available, simulation studies of large systems in three dimensions (3D) have become feasible. Hereby finite-size effects are reduced and sufficiently good resolution and statistics are ob- tained to allow for a detailed comparison with experimental data and analytical results. On the other hand, in simulation studies it may be difficult and very elaborate to establish the influence of variations in the model parameters on the physical properties. It is, therefore, of significant interest to establish analytical methods that may be used to analyze and explain these parameter dependences.

One of the main purposes of the present work is to formulate a theory for the diffuse scattering and the susceptibility $\chi(\mathbf{q})$ for a general class of lattice gas models. In the first approach a mean-field theory is established but it is developed further and utilized to calculate the effects of fluctuations on the phase diagram using the theoretical knowledge about $\chi(\mathbf{q})$ and the sum rule which relates $\chi(\mathbf{q})$ to the stoichiometry $x$. This is an alternative, and more systematic, way of including effects of correlations than provided by the cluster variation method (CVM) ${ }^{1-3}$ Our theory includes clusters of all sizes in a simple analytic form for any lattice gas Hamiltonian. The theory is useful for calculating the transition temperature $T_{c}$. However (like CVM), it is not aimed at discussing critical exponents and other critical properties.

The general mean-field matrix theory presented in this paper is applied to the ASYNNNI (anisotropic next-nearestneighbor interaction) model, which is a $2 \mathrm{D}$ anisotropic lattice gas model used to account for the oxygen ordering in the basal plane of the high-temperature superconductor $\mathrm{YBa}$ ${ }_{2} \mathrm{Cu}_{3} \mathrm{O}_{6+x} \cdot{ }^{4}$ In the standard form the ASYNNNI model, introduced by de Fontaine et al., ${ }^{5}$ contains concentrationand temperature-independent interaction parameters. It is 
fundamentally an anisotropic antiferromagnetic Ising model. When used in the case of $x=0.5$ for the variable oxygen stoichiometry it corresponds to having applied a finite magnetic field such that half the system is fully ferromagnetically polarized and the remaining half is in an antiferromagnetic state. It is a nontrivial model by which we can elucidate a number of principally interesting consequences of anisotropic interactions on the structure factor and it is furthermore of relevance for understanding the oxygen ordering in the $\mathrm{YBa}_{2} \mathrm{Cu}_{3} \mathrm{O}_{6+x}$ superconductor.

In the present work we have focused on the simple ASYNNNI model with concentration-independent shortrange interactions, and used the theory in order to describe its generic behavior. It is easy to include additional features, like longer-range interactions or $x$-dependent parameters, which may be needed in detailed comparisons with experiments. The theory is applicable also to the discussion of phase transition problems in, for example, alloys, which are often described by lattice gas models. The theory formulated for the susceptibility $\chi(\mathbf{q})$ and the scattering function $S(\mathbf{q})$ has been tested by comparing with accurate Monte Carlo simulation data performed for large systems. We have studied systems of size $256 \times 256 \times 16$ in order to achieve sufficiently high resolution in reciprocal space to permit a detailed analysis of the line shape. Such system sizes are comparable to the size of the twin domains found in $\mathrm{YBa}_{2} \mathrm{Cu}_{3} \mathrm{O}_{6+x}$ due to weak orthorhombic distortions in the oxygen-ordered phases. ${ }^{6}$

Another purpose of the present work is to investigate the consequences of adding to the ASYNNNI model an interaction in the third dimension, and use it in the region of the phase diagram where 2D studies have established good agreement with experiments. It is of particular interest to investigate the influence of a change in the oxygen stoichiometry around $x=0.5$ on both the short-range order and the line shape of the diffuse scattering. In this region the doublecell ortho-II structure is observed ${ }^{7-14}$ and there is evidence for a relation between the superconducting properties and the short-range oxygen order. ${ }^{4,15}$ This is the reason why the oxygen ordering phenomena in $\mathrm{YBa}_{2} \mathrm{Cu}_{3} \mathrm{O}_{6+x}$ have been studied intensively by neutron and $\mathrm{x}$-ray diffraction ${ }^{7,8,16-20,9}$ as well as other techniques. ${ }^{20,21}$ Computer simulations ${ }^{5,22-26}$ using the 2D ASYNNNI model have contributed to an understanding of an important part of the behavior in a certain range of temperatures $T$ and oxygen stoichiometry $x$. However, it has become clear that the ASYNNNI model is an idealized model, capable neither of describing thermodynamic properties like the chemical potential of oxygen faithfully in a large temperature interval ${ }^{23,26,8}$ nor of structural properties at low temperatures, ${ }^{11,12,14}$ or low oxygen stoichiometry, ${ }^{11,14,27}$ where additional long-range interactions including elastic forces seem to be needed to describe the observed partially ordered superstructures. ${ }^{28-31}$ Therefore, it is important to limit the use of the model to the region where it is valid. The simple 2D ASYNNNI model is particularly useful for describing the transition between a tetragonal disordered oxygen state and the orthorhombic ordered states for a single $\mathrm{CuO}_{x}$ plane. Thus, good agreement has been obtained for the experimental phase boundary and that found by Monte Carlo simulations and for the anisotropy of the in-plane peak widths of the structure factors. ${ }^{23}$ However, it is evident from the experiments ${ }^{7-9,14}$ that there is a significant correlation between the oxygen ordering in adjacent $\mathrm{CuO}_{x}$ planes. Hence there is a need for a three-dimensional model.

In this paper we have focused on equilibrium properties, and have found good agreement for $S(\mathbf{q})$ with the experiments in the disordered phase. In the ordered phase the 3D simulations show that it is very difficult to achieve an equilibrium one-domain state. This was not found in the corresponding 2D simulation studies. ${ }^{23,26}$ However, this observation is more in line with the experimental fact that it has so far been impossible to obtain long-range ordered superstructures in $\mathrm{YBa}_{2} \mathrm{Cu}_{3} \mathrm{O}_{6+x} .{ }^{8,32} \mathrm{We}$ have not undertaken a full study of the nonequilibrium behavior, but we will discuss a few results.

The paper is organized in the following way. In Sec. II we develop a mean-field (MF) matrix theory for calculating phase boundaries and structure factors of a general lattice gas Hamiltonian, and apply this theory to the 3D extension of the ASYNNNI model. In Sec. III we develop a theory for including the fluctuation effects on the phase diagram giving a functional dependence of the parameters in the model. Section IV contains the results of numerical Monte Carlo (MC) simulations and a comparison will be made with the analytical line shapes as well as recent experimental data. A summary and a conclusion are given in Sec. V.

\section{SUSCEPTIBILITIES AND STRUCTURE FACTORS}

In order to establish a systematic notation and the relations between the susceptibilities and structure factors, we consider a general class of lattice gas Hamiltonians of the form

$$
\begin{aligned}
\mathcal{H}= & -\frac{1}{2} \sum_{\alpha, \mathbf{r}_{\alpha}} \sum_{\beta, \mathbf{r}_{\beta}} J^{\alpha \beta}\left(\mathbf{r}_{\alpha}-\mathbf{r}_{\beta}\right) \sigma^{\alpha}\left(\mathbf{r}_{\alpha}\right) \sigma^{\beta}\left(\mathbf{r}_{\beta}\right) \\
& -\sum_{\alpha, \mathbf{r}_{\alpha}} \mu^{\alpha}\left(\mathbf{r}_{\alpha}\right) \sigma^{\alpha}\left(\mathbf{r}_{\alpha}\right)
\end{aligned}
$$

where in the sum $\Sigma_{\nu, \mathbf{r}_{\nu}}$ the vector $\mathbf{r}_{\nu}$ runs over all sites in the $\nu$ th sublattice, and $\nu$ runs over all sublattices $\nu=1, \ldots, N_{s}$. The interaction parameters between sublattices $\alpha$ and $\beta, J^{\alpha \beta}\left(\mathbf{r}_{\alpha}-\mathbf{r}_{\beta}\right)$, can conveniently be considered as matrix elements in a matrix $\mathbf{J}$. The site occupation variable $\sigma^{\alpha}\left(\mathbf{r}_{\alpha}\right)$ is equal to 1,0 depending on whether site $\mathbf{r}_{\alpha}$ is occupied or not. The chemical potential $\mu^{\alpha}\left(\mathbf{r}_{\alpha}\right)$ is in general inhomogeneous. The position vector $\mathbf{r}_{\alpha}$ can be written $\mathbf{r}_{\alpha}=\mathbf{R}+\mathbf{d}_{\alpha}$, where $\mathbf{R}=n_{1} \mathbf{a}_{1}+n_{2} \mathbf{a}_{2}+n_{3} \mathbf{a}_{3}$, and where $n_{1}$, $n_{2}$, and $n_{3}$ are integers. The Fourier transform of the matrix elements is given by

$$
J^{\alpha \beta}(\mathbf{q})=\sum_{\mathbf{R}} e^{i \mathbf{q} \cdot\left(\mathbf{R}+\mathbf{d}_{\alpha}-\mathbf{d}_{\beta}\right)} J^{\alpha \beta}\left(\mathbf{r}_{\alpha}-\mathbf{r}_{\beta}\right),
$$

which in an analogous manner may be arranged into the matrix $\mathbf{J}(\mathbf{q})$. In general we will calculate the Fourier transformation $A(\mathbf{q})$ of any quantity $A(\mathbf{r})$ by applying the transformation in Eq. (2). We separate the occupation number into two terms $\sigma^{\alpha}\left(\mathbf{r}_{\alpha}\right)=\left\langle\sigma^{\alpha}\left(\mathbf{r}_{\alpha}\right)\right\rangle+\delta \sigma^{\alpha}\left(\mathbf{r}_{\alpha}\right)$, where the first term is the thermal average occupation at the site $\mathbf{r}_{\alpha}$ and 
$\delta \sigma^{\alpha}\left(\mathbf{r}_{\alpha}\right)$ is the deviation from the average (similarly for $\beta)$. We denote the average value by $c_{\alpha}\left(\mathbf{r}_{\alpha}\right)=\left\langle\sigma^{\alpha}\left(\mathbf{r}_{\alpha}\right)\right\rangle$. The nonlocal susceptibility can then generally be expressed as

$$
\chi^{\alpha \beta}\left(\mathbf{r}_{\alpha}-\mathbf{r}_{\beta}\right)=\frac{1}{k_{B} T}\left\langle\delta \sigma^{\alpha}\left(\mathbf{r}_{\alpha}\right) \delta \sigma^{\beta}\left(\mathbf{r}_{\beta}\right)\right\rangle .
$$

The Fourier transform of $\chi^{\alpha \beta}\left(\mathbf{r}_{\alpha}-\mathbf{r}_{\beta}\right)$ is calculated as in Eq. (2) and is denoted by $\chi^{\alpha \beta}(\mathbf{q})$, which is conveniently arranged into the matrix $\boldsymbol{\chi}(\mathbf{q})$. It is shown in the Appendix, Eq. (A11), that the nonlocal susceptibility matrix $\boldsymbol{\chi}(\mathbf{q})$ in the mean-field (MF) approximation obeys a self-consistency relation relating the nonlocal susceptibility matrix to the local susceptibility matrix and the Fourier-transformed pair interaction matrix $\mathbf{J}(\mathbf{q})$ as follows:

$$
\boldsymbol{\chi}(\mathbf{q})=\left[(\stackrel{\circ}{\boldsymbol{\chi}})^{-1}-\mathbf{J}(\mathbf{q})\right]^{-1} .
$$

Here $\stackrel{\mathcal{X}}{ }$ is the local susceptibility matrix [see Eq. (A8)], with diagonal elements (all other matrix elements are zero)

$$
\stackrel{\circ}{\chi}_{\alpha}=\frac{1}{k_{B} T} c_{\alpha}\left(1-c_{\alpha}\right) \text {. }
$$

In the case where all sublattices are equivalent, which is generally the case in the disordered phase, there is no need for any subdivision into sublattices. Then the local susceptibility matrix obeys $\stackrel{\mathcal{\chi}}{=} \dot{\chi} \mathbf{1}$ and the average wave-vectordependent susceptibility (per site) becomes

$$
\chi_{\mathrm{av}}(\mathbf{q})=N_{s}^{-1} \sum_{\alpha \beta} \chi^{\alpha \beta}(\mathbf{q})=\left[(\stackrel{\circ}{\chi})^{-1}-\mathcal{J}(\mathbf{q})\right]^{-1},
$$

where $\mathcal{J}(\mathbf{q})$ is the Fourier transform of the total interaction that a given site is subject to, i.e., $\mathcal{J}(\mathbf{q})=N_{s}^{-1} \Sigma_{\alpha \beta} J^{\alpha \beta}(\mathbf{q})$. The structure factor $S(\mathbf{q})$ is generally calculated by separating the structure factor into two parts: $S(\mathbf{q})=S_{\text {Bragg }}(\mathbf{q})$ $+S_{\text {dif }}(\mathbf{q})$ (see Refs. 33-35), where

$$
\begin{aligned}
& S_{\text {Bragg }}(\mathbf{q})=\frac{1}{N_{\text {tot }}}\left|\sum_{\alpha, \mathbf{r}_{\alpha}} e^{i \mathbf{q} \cdot \mathbf{r}_{\alpha}}\left\langle\sigma^{\alpha}\left(\mathbf{r}_{\alpha}\right)\right\rangle b\right|^{2}, \\
& S_{\text {dif }}(\mathbf{q})=\frac{1}{N_{\text {tot }}}\left\langle\left|\sum_{\alpha, \mathbf{r}_{\alpha}} e^{i \mathbf{q} \cdot \mathbf{r}_{\alpha}} \delta \sigma^{\alpha}\left(\mathbf{r}_{\alpha}\right) b\right|^{2}\right\rangle .
\end{aligned}
$$

In Eq. (7), $N_{\text {tot }}$ is the total number of sites and all particles are assumed to have the same scattering length $b$. Combining Eqs. (3) and (7), the diffuse part of the structure factor can be expressed in terms of the nonlocal susceptibility matrix by

$$
S_{\mathrm{dif}}(\mathbf{q})=\frac{k_{B} T b^{2}}{N_{s}} \sum_{\alpha \beta} \chi^{\alpha \beta}(\mathbf{q}) .
$$

\section{A. Mean-field theory for the ASYNNNI model}

The modeling of the ordering of oxygen atoms in the $\mathrm{CuO}_{x}$ basal planes of $\mathrm{YBa}_{2} \mathrm{Cu}_{3} \mathrm{O}_{6+x}$, is done by the threedimensional extension of the ASYNNNI model. This anisotropic lattice gas model contains four pair interaction parameters $V_{1}, V_{2}, V_{3}$, and $V_{4}$ defined as follows (cf. Fig. 1). $V_{1}$ is the nearest neighbor (NN) repulsion between pairs of oxygen atoms. $V_{2}$ is the next nearest neighbor $(\mathrm{NNN})$ attrac-

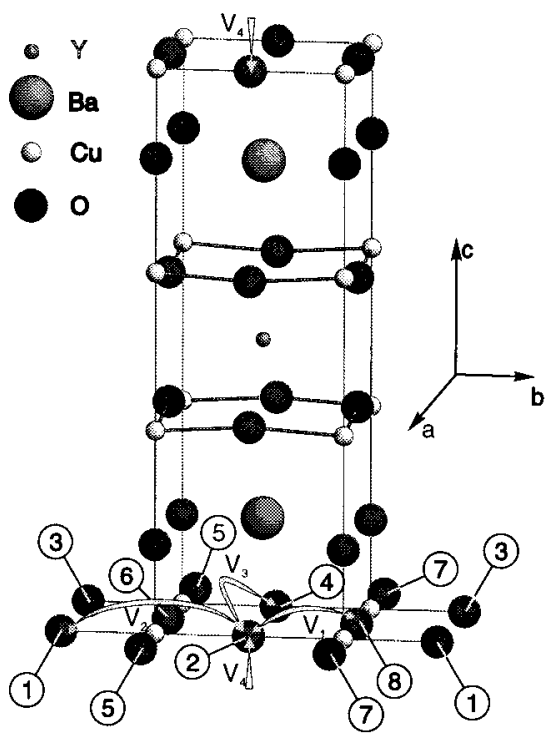

FIG. 1. The crystallographic structure of $\mathrm{YBa}_{2} \mathrm{Cu}_{3} \mathrm{O}_{6+x}$ with a unit cell indicated by the solid lines. All oxygen sites assume perovskite positions located halfway between the copper atoms along the square edges. The variable oxygen stoichiometry refer to the $O 1$ and $O 5$ sites in the basal planes, which are separated by $|\mathbf{c}|=11.7 \AA$ along the vertical $c$ axis. The $O 1$ and $O 5$ sites are represented by sublattices $1, \ldots, 8$ used in defining the mean-field Hamiltonian. The numbering convention derives from Aukrust et al. (Ref. 39). The $a$ axis is along the (1)-(3) sites and the $b$ axis is along the (5)-(7) sites. The oxygen atoms prefer to order in chains, forming subsystems as discussed in the text. The oxygen pair interaction parameters $V_{1}, V_{2}, V_{3}$, and $V_{4}$ are defined as follows: $V_{1}$ is the nearest neighbor repulsion, $V_{2}$ is the next nearest neighbor attraction between pairs bridged by $\mathrm{Cu}, V_{3}$ is the next nearest neighbor repulsion between pairs not bridged by $\mathrm{Cu}$, and $V_{4}$ is the nearest neighbor attraction between pairs on adjacent basal planes.

tive interaction between pairs of oxygen atoms which are bridged by a copper atom ( $\mathrm{NNNCu}$ ), while $V_{3}$ a repulsive interaction between oxygen atoms which are not bridged by a copper atom (NNNV). The interplane coupling is introduced by an attractive interaction $V_{4}$ between pairs of oxygen atoms located vertically above and below each other on adjacent basal planes (NNPl). This leads to the Hamiltonian

$$
\begin{aligned}
\mathcal{H}= & -V_{1} \sum_{\left\langle\mathbf{r r}^{\prime}\right\rangle}^{\mathrm{NN}} \sigma(\mathbf{r}) \sigma\left(\mathbf{r}^{\prime}\right)-V_{2} \sum_{\left\langle\mathbf{r r}^{\prime}\right\rangle}^{\mathrm{NNNCu}} \sigma(\mathbf{r}) \sigma\left(\mathbf{r}^{\prime}\right) \\
& -V_{3} \sum_{\left\langle\mathbf{r r}^{\prime}\right\rangle}^{\mathrm{NNNV}} \sigma(\mathbf{r}) \sigma\left(\mathbf{r}^{\prime}\right)-V_{4} \sum_{\left\langle\mathbf{r r}^{\prime}\right\rangle}^{\mathrm{NNP1}} \sigma(\mathbf{r}) \sigma\left(\mathbf{r}^{\prime}\right)-\mu \sum_{\mathbf{r}} \sigma(\mathbf{r}),
\end{aligned}
$$

where the sums run over all oxygen pairs $\left\langle\mathbf{r r}^{\prime}\right\rangle$, which are nearest neighbor ( $\mathrm{NN}$ and $\mathrm{NNPl}$ ) or next nearest neighbors ( $\mathrm{NNNV}$ and $\mathrm{NNNCu}$ ), respectively. The relative magnitude of the interaction parameters $V_{1}, V_{2}$, and $V_{3}$ has been established by a comparison between experimental data and 2D simulation results, ${ }^{4,23,36,37}$ while the additional interplane coupling $V_{4}$ has been adopted from Ref. 38, 


$$
\left(V_{1}, V_{2}, V_{3}, V_{4}\right)=\left(-V_{0}, 0.36 V_{0},-0.12 V_{0}, 0.02 V_{0}\right),
$$

with $V_{0} / k_{B}=4490 \mathrm{~K}$. The ASYNNNI model Hamiltonian can be brought into the form of Eq. (1) by using the $N_{s}=8$ sublattices introduced by Aukrust et al. ${ }^{39}$ (see Fig. 1). This gives rise to the interaction parameters $J^{\alpha \beta}\left(\mathbf{r}_{\alpha}-\mathbf{r}_{\beta}\right)$, with $\alpha, \beta=1, \ldots, 8$, between sublattice $\alpha$ and sublattice $\beta$. The physical relevant interaction parameters for $\mathrm{YBa}_{2} \mathrm{Cu}_{3} \mathrm{O}_{6+x}$ are $V_{2}>0>V_{3} \gg V_{1}$ and $V_{4}>0$ which stabilize the experimentally observed ortho-I $(x=1)$ and the ortho-II $\left(x=\frac{1}{2}\right)$ phases. The ground state is easily determined from Eq. (A1) by considering the minimum of $\left\langle\mathcal{H}^{\mathrm{MF}}\right\rangle$ subject to the constraint that the average concentration satisfies $c_{\mathrm{av}}$ $\equiv N_{s}^{-1} \Sigma_{\alpha} c_{\alpha}=x / 2$. Due to the symmetries in the Hamiltonian, the concentrations satisfy $\left(c_{1}=c_{2} \equiv c_{a}\right)$, $\left(c_{3}=c_{4} \equiv c_{b}\right),\left(c_{5}=c_{6} \equiv c_{c}\right)$, and $\left(c_{7}=c_{8} \equiv c_{d}\right)$, where we have introduced four concentrations $c_{a}, c_{b}, c_{c}$, and $c_{d}$. Finite values for given $c_{\alpha}$ correspond to occupied chains of oxygen atoms, as can be seen from Fig. 1. We shall denote partial occupancy in terms of subsystems of the full $1, \ldots, 8$ system.

Consider first the ortho-II phase at $x=\frac{1}{2}$. There are four possible domains, which are occupied 1-2 chains, while the remaining sites are empty $\left(c_{a}=1\right)$; occupied 3-4 chains, while the remaining sites are empty $\left(c_{b}=1\right)$; and analogously for the perpendicular 5-6 chains $\left(c_{c}=1\right)$ and the 7-8 chains $\left(c_{d}=1\right)$; see Fig. 1 . In the pure ortho-I phase, at $x=1$ there are two possible domain types. These are occu- pied 1-2 and 3-4 chains $\left(c_{a}=c_{b}=1\right)$ with the remaining sites being empty and occupied perpendicular 5-6 and 7-8 chains $\left(c_{c}=c_{d}=1\right)$ with the remaining sites empty. At low, finite temperatures in the ortho-II phase, the domain consisting of predominantly occupied $1-2$ chains $\left(c_{a}=1\right)$ has in addition a smaller thermal occupation of both the parallel 3-4 chains $\left(c_{b} \neq 0\right)$ and of the perpendicular 5-6 and 7-8 chains $\left(c_{c}=c_{d} \neq 0\right)$. Upon increasing the temperature, we may enter the ortho-I phase where $c_{a}=c_{b}$ and $c_{c}=c_{d}$. Consequently, the phase boundary separating the ortho-II and the ortho-I phases can be determined as the temperature at which the equality $c_{a}=c_{b}$ becomes valid. If we increase the temperature even further, all the sublattices eventually become equally populated, $c_{a}=c_{b}=c_{c}=c_{d}$, and we enter the tetragonal phase. Similarly, the phase boundary separating the ortho-I phase and the tetragonal phase can be determined from this equality. In the case of a continuous transition the phase boundaries can be obtained much more simply from the condition of a diverging diffuse structure factor or susceptibility.

The Fourier transform ${ }^{40}$ of the interaction matrix $\mathbf{J}$ can be written in terms of the interaction parameters in Eq. (9) as

$$
\mathbf{J}(\mathbf{q})=\left(\begin{array}{cc}
\mathbf{A}(\mathbf{q}) & \mathbf{B}(\mathbf{q}) \\
\mathbf{B}^{\dagger}(\mathbf{q}) & \mathbf{A}(\hat{\mathbf{q}})
\end{array}\right),
$$

where $\mathbf{A}(\mathbf{q})$ and $\mathbf{B}(\mathbf{q})$ are $4 \times 4$ matrices and $\mathbf{A}(\mathbf{q})$ is given by

$$
\mathbf{A}(\mathbf{q})=\left(\begin{array}{cccc}
2 V_{4} \cos \left(q_{z}\right) & 2 V_{2} \cos \left(q_{y}\right) & 2 V_{3} \cos \left(q_{x}\right) & 0 \\
2 V_{2} \cos \left(q_{y}\right) & 2 V_{4} \cos \left(q_{z}\right) & 0 & 2 V_{3} \cos \left(q_{x}\right) \\
2 V_{3} \cos \left(q_{x}\right) & 0 & 2 V_{4} \cos \left(q_{z}\right) & 2 V_{2} \cos \left(q_{y}\right) \\
0 & 2 V_{3} \cos \left(q_{x}\right) & 2 V_{2} \cos \left(q_{y}\right) & 2 V_{4} \cos \left(q_{z}\right)
\end{array}\right)
$$

The interaction matrix $\mathbf{B}(\mathbf{q})$ can be written, using the phase factors $p=e^{i\left(q_{x}+q_{y}\right) / 2}$ and $m=e^{i\left(q_{x}-q_{y}\right) / 2}$, as

$$
\mathbf{B}(\mathbf{q})=V_{1}\left(\begin{array}{cccc}
p & m & m^{*} & p^{*} \\
m^{*} & p^{*} & p & m \\
m & p & p^{*} & m^{*} \\
p^{*} & m^{*} & m & p
\end{array}\right)
$$

To describe the perpendicular subsystems, we have introduced the vector $\hat{\mathbf{q}}=\left(q_{y}, q_{x}, q_{z}\right)$ related to $\mathbf{q}=\left(q_{x}, q_{y}, q_{z}\right)$ by a rotation of $90^{\circ}$. In the following we will, using mean-field theory, discuss the pertinent features of the structure factor which subsequently is studied by Monte Carlo simulations. The MF results are important in order to gain insight into the multidimensional parameter space and to derive suitable functional forms to which experimental or numerical data for $S_{\text {dif }}(\mathbf{q})$ may be fitted. These functional forms correctly include a number of important geometrical features, but fluctuation effects are of course not included. It is convenient to introduce the susceptibilities of subsystems, corresponding to using all the symmetries of the oxygen-ordered phases, where all single sites within the subsystem have the same oxygen occupancy and consequently the same local susceptibility $\dot{\chi}$. Consider first the subsystem consisting of sublattices 1,2,3,4 (ortho-I symmetry). The average susceptibility for this subsystem, $\chi_{14}(\dot{\chi}, \mathbf{q})$, can be calculated using Eq. (4) where the $4 \times 4$ matrix $\mathbf{A}(\mathbf{q})$, Eq. (12), is to be used as the interaction matrix $\mathbf{J}(\mathbf{q})$. Since all sites in this case are equivalent, we obtain according to Eq. (6)

$\chi_{14}(\stackrel{\circ}{\chi}, \mathbf{q})$

$$
=\frac{1}{(\stackrel{\circ}{\chi})^{-1}-2 V_{3} \cos \left(q_{x}\right)-2 V_{2} \cos \left(q_{y}\right)-2 V_{4} \cos \left(q_{z}\right)} .
$$

Analogously, the susceptibility for the perpendicular subsystem of the ortho-I symmetry is given by $\chi_{14}(\stackrel{\circ}{\chi}, \hat{\mathbf{q}})$. In the 
ortho-I phase we have the situation $c_{a}=c_{b} \neq c_{c}=c_{d}$, and we denote the local susceptibilities $\stackrel{\circ}{\chi}_{a}$ and $\dot{\mathcal{X}}_{d}$ for the two subsystems, respectively. The structure factor can be calculated directly from Eqs. (4) and (8) or alternatively by combining the susceptibilities $\chi_{14}\left(\dot{\chi}_{a}, \mathbf{q}\right)$, and $\chi_{14}\left(\stackrel{\circ}{\chi}_{d}, \hat{\mathbf{q}}\right)$ from the two subsystems $1,2,3,4$ and 5,6,7,8 according to Eq. (A12). The coupling matrix is $\mathbf{V}(\mathbf{q})=\mathbf{B}(\mathbf{q})$ and consequently $\bar{V}(\mathbf{q})$ $=V_{1}\left(p+p^{*}+m+m^{*}\right)$. We obtain

$$
\begin{aligned}
S_{\mathrm{dif}}(\mathbf{q})= & k_{B} T b^{2} \\
& \times \frac{\frac{1}{2}\left\{\chi_{14}^{-1}\left(\stackrel{\circ}{\alpha}_{a}, \mathbf{q}\right)+\chi_{14}^{-1}\left(\stackrel{\circ}{\alpha}_{d}, \hat{\mathbf{q}}\right)\right\}+4 V_{1} \cos \left(q_{x} / 2\right) \cos \left(q_{y} / 2\right)}{\chi_{14}^{-1}\left(\dot{\circ}_{a}, \mathbf{q}\right) \chi_{14}^{-1}\left(\stackrel{\circ}{\chi}_{d}, \hat{\mathbf{q}}\right)-16 V_{1}^{2} \cos ^{2}\left(q_{x} / 2\right) \cos ^{2}\left(q_{y} / 2\right)} .
\end{aligned}
$$

In the tetragonal phase the two local susceptibilities are equal,

$$
\dot{\circ}_{a}=\stackrel{\circ}{\chi}_{d}=\frac{1}{k_{B} T} \frac{x}{2}\left(1-\frac{x}{2}\right),
$$

and $S_{\text {dif }}(\mathbf{q})$ from Eq. (15) is isotropic due to the invariance under the exchange $\mathbf{q} \leftrightarrow \hat{\mathbf{q}}$. We may use Eq. (15) to determine the phase boundary between the ortho-I and the tetragonal phases by determining the temperatures of divergence of $S_{\text {dif }}\left(\mathbf{Q}_{\mathrm{I}}\right)$, where $\mathbf{Q}_{\mathrm{I}}=(2 \pi, 0,0)$ is the characteristic ortho-I reflection. Direct evaluation using Eqs. (5) and (14) yields

$$
k_{B} T_{c}^{\mathrm{MF}}(x)=x\left(1-\frac{x}{2}\right)\left(-2 V_{1}+V_{2}+V_{3}+V_{4}\right) .
$$

In the ortho-I phase, the local susceptibilities of the two subsystems are different, $\dot{\chi}_{a} \neq \dot{\chi}_{d}$. We can without loss of generality assume a predominant occupation of the 1-4 subsystem $\left(c_{a} \gg c_{d}\right)$ constrained by the condition $c_{a}+c_{d}=x$. It follows from Eq. (A4) to first order in $\epsilon$, where $\epsilon=e^{4 V_{1} c_{a} / k_{B} T}$, that $c_{d}=\epsilon /(1+\epsilon)$. Due to the strong nearest neighbor repulsion $V_{1}\left(V_{1}<0\right)$, it follows that $\epsilon$ becomes exponentially small as we reduce $k_{B} T$. According to Eq. (5), $\dot{\varkappa}_{d}=\epsilon / k_{B} T$ and we may use $\dot{\circ}_{d}$ as an expansion parameter in an expansion for Eq. (15). To leading order in $\epsilon$ we obtain

$$
\begin{aligned}
S_{\mathrm{dif}}(\mathbf{q})= & k_{B} T b^{2} \\
& \times \chi_{14}\left(\stackrel{\circ}{\chi}_{a}, \mathbf{q}\right) \frac{1}{2}\left[\frac{1+4 V_{1} \stackrel{\circ}{\chi}_{d} \cos \left(q_{x} / 2\right) \cos \left(q_{y} / 2\right)}{1-16 V_{1}^{2} \stackrel{\circ}{\chi}_{d} \chi_{14}\left(\stackrel{\circ}{\chi}_{a}, \mathbf{q}\right) \cos ^{2}\left(q_{x} / 2\right) \cos ^{2}\left(q_{y} / 2\right)}\right] .
\end{aligned}
$$

Anticipating the phase transition to the ortho-II phase with the divergence at the characteristic wave vector $\mathbf{Q}_{\mathrm{II}}=(\pi, 0,0)$ we may expand $S_{\mathrm{dif}}(\mathbf{q})$ around $\mathbf{Q}_{\mathrm{II}}$ to second order in $\mathbf{q}$, yielding

$$
\begin{aligned}
S_{\mathrm{dif}}\left(\mathbf{Q}_{\mathrm{II}}+\mathbf{q}\right)= & k_{B} T b^{2} \\
& \times \frac{1}{2}\left[\frac{1-2 V_{1} \stackrel{\circ}{d}_{d} q_{x}}{\left(\chi_{a}\right)^{-1}+V_{2} q_{y}^{2}-\left(V_{3}+4 V_{1}^{2} \stackrel{\circ}{d}_{d}\right) q_{x}^{2}+V_{4} q_{z}^{2}}\right],
\end{aligned}
$$

where we have introduced the diverging susceptibility $\chi_{a} \equiv \chi_{14}\left(\stackrel{\circ}{\chi}_{a}, \mathbf{Q}_{\mathrm{II}}\right)=\left[\stackrel{\circ}{\chi}_{a}^{-1}+2 V_{3}-2 V_{2}-2 V_{4}\right]^{-1}$. If instead we have considered the alternative ordered domain with the 5-8 chains occupied and the 1-4 chains being empty, the result is identical except that the peak occurs at $\hat{\mathbf{Q}}_{\mathrm{II}}$ and that $q_{x}$ and $q_{y}$ in Eq. (17) are interchanged. The mean-field critical temperature $T_{c}^{\mathrm{MF}}(x)$ for the ortho-I-ortho-II transition is determined from Eq. (18) as the temperature at which $S_{\text {dif }}\left(\mathbf{Q}_{\mathrm{II}}\right)$ diverge $\left(\chi_{a}^{-1}=0\right)$. We obtain to leading order in $\epsilon$

$$
k_{B} T_{c}^{\mathrm{MF}}(x)=2(x-\epsilon)(1+\epsilon-x)\left(V_{2}+V_{4}-V_{3}\right) .
$$

The resulting mean-field phase diagram is not expected to be accurate with respect to the onset of long-range order (LRO), i.e., the positions of the phase boundaries. The effects of fluctuations will modify it strongly as discussed in Sec. III. However, it has previously been found ${ }^{41}$ that the mean-field phase boundaries nonetheless are very useful since they indicate where the short-range order (SRO) becomes significant.

According to Eq. (18) the line shape of $S_{\text {dif }}(\mathbf{q})$ near a Bragg point, $\mathbf{Q}_{\mathrm{II}}$, is essentially a Lorentzian, with anisotropic full width at half maxima (FWHM). This is slightly modulated by a multiplicative "background" function $1-2 V_{1} \stackrel{\circ}{\chi}_{d} q_{x}$. The modulating function is linear in $q_{x}$, giving rise to a shift in the ortho-II superstructure reflection by $\Delta Q_{x}$. To first order in $\epsilon$ the shift is $\Delta Q_{x}=\epsilon\left|V_{1}\right| /\left(2 k_{B} T\left|V_{3}\right| \chi_{a}\right)$. Because of the dependence on $\chi_{a}, \Delta Q_{x}$ vanishes at $T_{c}^{\mathrm{MF}}$ as given in Eq. (19). Therefore, the Bragg peak develops exactly at $\mathbf{Q}_{\mathrm{II}}$. However, above $T_{c}^{\mathrm{MF}}$, within the ortho-I phase, a shift of the peak is predicted. This shift is also present when ortho-I LRO is replaced by ortho-I SRO and $\chi_{a}$ is replaced by the exact susceptibility, which is diverging at the true $T_{c}<T_{c}^{\mathrm{MF}}$. In the region $T_{c}<T<T_{c}^{\mathrm{MF}}$ the 
LRO is replaced by a SRO in which there simultaneously exist finite domains of populated 1-4 and 5-8 subsystems. The ortho-I Bragg peak is therefore broadened to an anisotropic Lorentzian, reflecting the spatial extent of the ortho-I domains. Below the transition temperature to the ortho-II structure, these domains are further subdivided into the corresponding ortho-II domains, with the chains in the given directions. This gives rise to the anisotropic ortho-II (SRO) peaks at $\mathbf{Q}_{\mathrm{II}}$ and $\hat{\mathbf{Q}}_{\mathrm{II}}$. From Eq. (17) we can determine the ratios of the anisotropies in the FWHM, $\Delta k(T) / \Delta h(T)$ and $\Delta l(T) / \Delta h(T)$. To first order in $\epsilon$ we obtain $\Delta k(T) /$ $\Delta h(T)=\sqrt{\left(\left|V_{3}\right|+4 V_{1}^{2}{ }^{\circ}{ }_{d}\right) /\left|V_{2}\right|}$. Analogously we obtain $\Delta l(T) / \Delta h(T)=\sqrt{\left(\left|V_{3}\right|+4 V_{1}^{2} \dot{\circ}_{d}\right) /\left|V_{4}\right|}$. This allows for a determination of the interaction parameters from a measurement of the relative widths of the structure factor. The widths $\Delta h(T), \Delta k(T)$, and $\Delta l(T)$ should scale with the reduced temperature as a power law $\left|T-T_{c}\right|^{\nu}$, with $\nu^{\mathrm{MF}}=\frac{1}{2}$. It is important to note, that according to mean-field theory the anisotropies $\Delta k / \Delta h$ and $\Delta l / \Delta h$ are independent of temperature and oxygen stoichiometry $x$, when the influence of $\dot{\chi}_{d}$ can be neglected.

In the same fashion, we can discuss the structure factor in the ortho-II phase. Since $\epsilon$ is exponentially small at low $T$, we will take $\epsilon=0$. Consider the subsystem consisting of only sublattices 1,2 (ortho-II symmetry). The susceptibility for this system, $\chi_{12}(\chi, \mathbf{q})$, can be found in an analogous way from Eq. (11) by using Eq. (6). We obtain

$$
\chi_{12}(\stackrel{\circ}{\chi}, \mathbf{q})=\frac{1}{(\stackrel{\circ}{\chi})^{-1}-2 V_{2} \cos \left(q_{y}\right)-2 V_{4} \cos \left(q_{z}\right)} .
$$

The susceptibility for the 3-4 subsystem is identical to that of the 1-2 subsystem and is therefore also given by Eq. (20). The oxygen chains for the 5-6 and the 7-8 subsystem are rotated by $90^{\circ}$ and thus the susceptibility is given by $\chi_{12}(\dot{\chi}, \hat{\mathbf{q}})$. The structure factor in the ortho-II phase can be calculated by combining the susceptibilities $\chi_{12}\left(\dot{\chi}_{a}, \mathbf{q}\right)$, and $\chi_{12}\left(\dot{\chi}_{b}, \mathbf{q}\right)$ from the two subsystems 1,2 and 3,4 according to Eq. (A12), where the average coupling is given by $\bar{V}(\mathbf{q})=2 V_{3} \cos \left(q_{x}\right)$. We obtain

$$
S_{\mathrm{dif}}(\mathbf{q})=k_{B} T b^{2} \frac{\frac{1}{2}\left\{\chi_{12}^{-1}\left(\dot{\circ}_{a}, \mathbf{q}\right)+\chi_{12}^{-1}\left(\stackrel{\circ}{\chi}_{b}, \mathbf{q}\right)\right\}+2 V_{3} \cos \left(q_{x}\right)}{\chi_{12}^{-1}\left(\dot{\circ}_{a}, \mathbf{q}\right) \chi_{12}^{-1}\left(\dot{\circ}_{b}, \mathbf{q}\right)-4 V_{3}^{2} \cos ^{2}\left(q_{x}\right)} .
$$

The corresponding expansion of $S_{\text {dif }}(\mathbf{q})$ around $\mathbf{Q}_{\text {II }}$ gives an anisotropic Lorentzian. There is no shift in the Bragg position in this case, since there is no term linear in $q_{x}$. The anisotropies are determined by $\Delta k(T) / \Delta h(T)$ $=\gamma \sqrt{\left|V_{3}\right| /\left|V_{2}\right|}$ and $\Delta l(T) / \Delta h(T)=\gamma \sqrt{\left|V_{3}\right| /\left|V_{4}\right|}$, where $\gamma=\sqrt{2 \dot{\chi}_{a} \dot{\circ}_{b} /\left(\dot{\chi}_{a}^{2}+\dot{\chi}_{b}^{2}\right)}$. This completes the mean-field calculation of the line shapes. These line shapes include very important geometrical effects (like the shift in the peak position) and give a representation in the entire reciprocal space. As will be shown, the inclusion of these features is pertinent for obtaining an understanding of the Monte Carlo results.

\section{INCLUSION OF FLUCTUATION EFFECTS}

It is well known that mean field-theory predicts a too high transition temperature because the fluctuation effects have been neglected. That implies that $T_{c}^{\mathrm{MF}} \gg T_{c}$ in particular for low-dimensional systems, where the fluctuations are large. On the other hand, the MF functional form of the wavevector-dependent susceptibility is expected to be quite accurate above $T_{c}$. This will in fact be demonstrated below, using the Monte Carlo simulations. The average wave-vectordependent susceptibility $\chi_{\text {av }}(\mathbf{q})$ in Eq. (6) satisfies the sum rule $N_{\text {tot }}^{-1} \Sigma_{\mathbf{q}} \chi_{\text {av }}(\mathbf{q})=\left(k_{B} T\right)^{-1} c_{\text {av }}\left(1-c_{\text {av }}\right)$, where $N_{\text {tot }}$ is the total number of sites and $c_{\mathrm{av}}$ is the average concentration. According to Eq. (6), $T_{c}^{\mathrm{MF}}=\mathcal{J}(\mathbf{Q}) c_{\mathrm{av}}\left(1-c_{\mathrm{av}}\right)$. By replacing the sum $N_{\text {tot }}^{-1} \Sigma_{\mathbf{q}}$ by the volume integral over the Brillouin zone, the sum rule can be written in the form ${ }^{42,43}$

$$
\frac{1}{(2 \pi)^{3}} \int_{0}^{2 \pi} d q_{x} \int_{0}^{2 \pi} d q_{y} \int_{0}^{2 \pi} d q_{z} \frac{1}{R-\mathcal{J}(\mathbf{q}) / \mathcal{J}(\mathbf{Q})}=\frac{T_{c}^{\mathrm{MF}}}{T},
$$

where we have used that $\chi_{\text {av }}(\mathbf{q})$ in the disordered phase has the form Eq. (6). The principle behind the approximation is to assume that $R$ can be generalized beyond the MF value to represent the true local inverse susceptibility. Then $R$ can be treated as an independent variable and Eq. (22) used to determine $T$ for a given $R$. For continuous variables this approach is called the spherical approximation. ${ }^{42}$ When used in the Heisenberg model, one can obtain a very accurate estimate for the correct $T_{c}$ by determining it as the temperature at which Eq. (22) is fulfilled when $\chi_{\text {av }}(\mathbf{Q})$ diverges, i.e., when $R \rightarrow 1{ }^{42}$ One obtains, for example, the correct dependence on the length of the spin in Heisenberg spin systems, and the correct dependence of the spatial dimensionality $D$, in the sense that $T_{c}=0$ for $D \leqslant 2 .{ }^{42}$ The spherical approximation has not previously been used for Ising-like models. For the Ising model the dynamics is stochastic and the use of Eq. (22) requires modification due to the fact that it always costs a finite energy to flip a spin or in our case to add or remove an oxygen atom. A similar energy gap arises in the case of small Heisenberg clusters. ${ }^{43}$ Therefore we introduce a gap at $\mathbf{q}=\mathbf{Q}$, and determine $T_{c}$ as the temperature at which $R=1+\delta$, with a small $\delta .{ }^{44}$ An alternative method of including correlation effects on the phase diagram is the CVM theory. ${ }^{1-3}$ The result of the CVM theory depends on how large clusters are included in the calculation. In the present theory clusters of all sizes are automatically included via the complete sum over the entire reciprocal space.

We shall here calculate the fluctuation-corrected $T_{c}$ using Eq. (22) for the ortho-I-ortho-II transition by applying the sum rule to the susceptibility $\chi_{14}(\stackrel{\circ}{\chi}, \mathbf{q})$, with $\mathcal{J}(\mathbf{q})$ $=2\left[V_{2} \cos \left(q_{x}\right)+V_{3} \cos \left(q_{y}\right)+V_{4} \cos \left(q_{z}\right)\right]$. This requires solving the integral

$$
G(t)=\int_{0}^{2 \pi} d x \int_{0}^{2 \pi} d y \int_{0}^{2 \pi} d z \frac{1}{t-\alpha \cos x-\beta \cos y-\gamma \cos z} .
$$

Thus we need a generalization of the isotropic Watson integral. ${ }^{45}$ By applying the method of Morita and Horiguchi ${ }^{46}$ the integral can be shown to be given by

$$
G(t)=\frac{8 \pi}{\sqrt{\gamma \beta}} \int_{0}^{\pi} d x k K\left(k^{2}\right)
$$




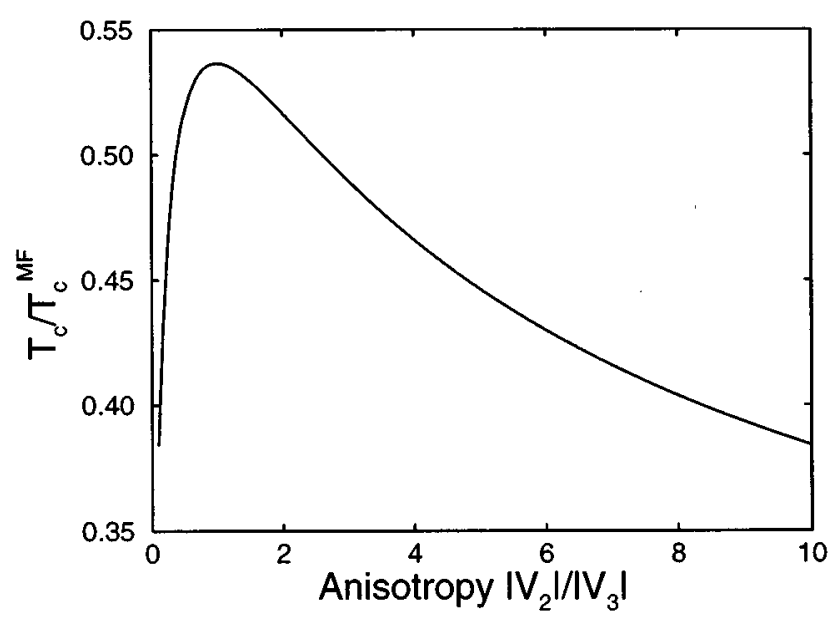

FIG. 2. The ortho-I-ortho-II transition temperature $T_{c} / T_{c}^{\mathrm{MF}}$ at $x \sim \frac{1}{2}$ plotted versus the inplane anisotropy $\left|V_{2}\right| /\left|V_{3}\right|$. The results are based on numerical solution of Eq. (22), for the parameter values $V_{1}=-V_{0}, V_{2}=0.36 V_{0}, V_{3}=-0.12 V_{0}, T_{c}^{\mathrm{MF}}=0.24 V_{0} / k_{B}$.

where

$$
k=\left[\frac{4 \beta \gamma}{(t-\alpha \cos x)^{2}-(\beta-\gamma)^{2}}\right]^{1 / 2},
$$

and $K(k)$ is the complete elliptic integral of the first kind; see Ref. 47. The sum rule allows us to investigate the influence of parameters in the model. First, we consider the dependence on $T_{c}$ of the in-plane anisotropy, $\left|V_{2}\right| /\left|V_{3}\right|$, of the ortho-I-ortho-II phase transition. By using Eqs. (22) and (24) we estimate $R=1.02$ in order to obtain the correct transition temperature for the 2D ASYNNNI model (at $x=\frac{1}{2}$ ), as determined from Monte Carlo simulations. ${ }^{23}$ This is shown in Fig. 2. For the isotropic case we obtain $T_{c}$ $=2.144 V_{0} / k_{B}$. This can be compared with the exact $T_{c}=2.268 V_{0} / k_{B}$ for the nearest neighbor Ising model. For the cluster variation method the corresponding results are ${ }^{48}$ for the pair approximation $T_{c}=2.885 V_{0} / k_{B}$ and for the fourpoint-five-point approximation $T_{c}=2.359 V_{0} / k_{B}$. As we

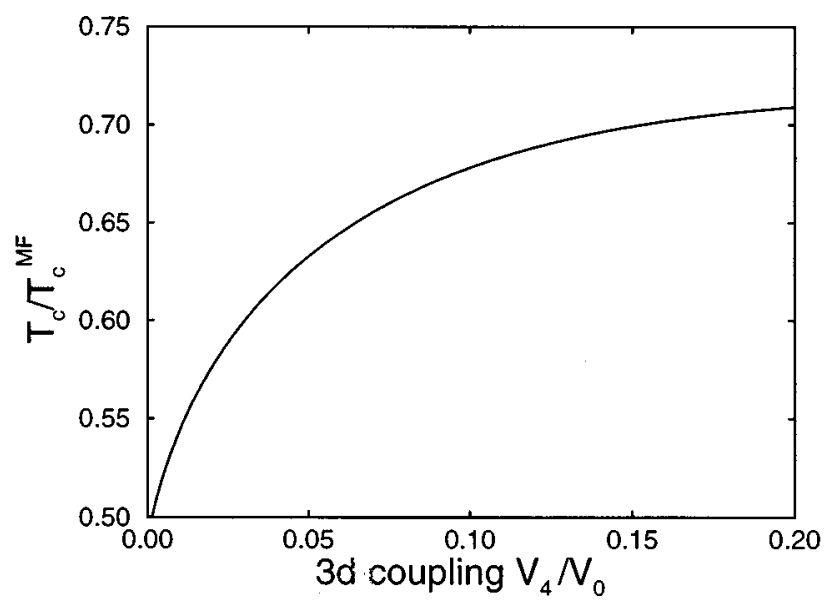

FIG. 3. The ortho-I-ortho-II transition temperature $T_{c} / T_{c}^{\mathrm{MF}}$ at $x \sim \frac{1}{2}$ plotted versus the interplane interaction parameter $V_{4} / V_{0}$. The results are obtained as in Fig. 2. move away from the isotropic case the model attains some $1 \mathrm{D}$ character and $T_{c}$ decreases. Next we consider the dependence of $T_{c}$ on the interplane coupling, $V_{4} / V_{0}$. We have in Fig. 3 calculated $T_{c}\left(V_{4}\right) / T_{c}^{\mathrm{MF}}$. This result shows that the introduction of even a very small interplane coupling $V_{4}$ leads to a dramatic increase in $T_{c}$, and much larger than could be expected from mean-field theory [cf. Eq. (14)]. This is because the two-dimensional Ising model is a case of marginal LRO. ${ }^{44}$ For the isotropic case, $\left|V_{2}\right|=\left|V_{3}\right|=\left|V_{4}\right|$, we find $T_{c}=4.487 V_{0} / k_{B}$, which can be compared with $T_{c}=4.511 V_{0} / k_{B}$ obtained from high-accuracy Monte Carlo studies. ${ }^{49}$ In Sec. IV we report on our Monte Carlo simulation results using the $3 \mathrm{D}$ ASYNNNI model with $V_{4}=0.02\left|V_{1}\right|$. The $T_{c}$ obtained from the Monte Carlo simulation is in excellent agreement with the estimate from the sum rule. Therefore Fig. 3 allows us to predict $T_{c}\left(V_{4}\right)$ reliably for other values of the interplane coupling parameter.

We now turn to a discussion of the $x$ dependence of $T_{c}$. In the dilute limit, $x<x_{p}$, where $x_{p}$ is the percolation threshold ( $x_{p} \approx 0.3$ for a monodomain of ortho-II), the LRO is replaced by SRO even at $T=0$, since for these low concentrations the particles cannot arrange themselves into ortho-I or ortho-II structures. The same arguments hold for $x>1-x_{p}$, so that for the ortho-II phase, LRO is replaced by SRO. Thus, when $x \rightarrow 1$ the ortho-II structure is not stable. In this limit the small number of vacancies will at $T=0$ arrange themselves in long chains. If there are no long-range interactions, these chains will be randomly distributed and constitute a so-called Magneli phase. ${ }^{50,51}$ If there are long-range interactions, other phases may appear. Recently, there has been found experimental evidence for the presence of SRO of the ortho-III symmetry at low temperatures and $x=0.77 .{ }^{32}$ This phase consists of two full chains with the third missing, etc. Theoretically, such phases have been considered by Ceder et $a l .{ }^{30}$ and by Zubkus et $a l .{ }^{31}$ However, since the ordering kinetics at low temperature is very slow, the higher-order modulated phases are difficult to form and therefore to observe in both experiments and, in particular, in Monte Carlo simulations. Consequently, we shall not discuss the $x \rightarrow 0$ and $x \rightarrow 1$ regions. For $x \sim \frac{1}{2}$, on the other hand, we expect the ASYNNNI model to include most of the pertinent interactions needed to describe the high-temperature behavior. Therefore, the value of $T_{c}$ calculated using Eq. (22) and Eq. (24) is predicted to have the same $x$ dependence as $T_{c}^{\mathrm{MF}}$, i.e.,

$$
T_{c}\left(x^{\prime}\right)=T_{c}\left(x=\frac{1}{2}\right)\left[1-4\left(x^{\prime}-\epsilon\right)^{2}\right],
$$

where $x^{\prime}=x-\frac{1}{2}$. Accordingly, a parabolic dependence on $x^{\prime}$ is predicted with a maximum shifted towards $x>\frac{1}{2}$. Since $\epsilon$ increases with temperature, the shift is predicted to be larger if $T_{c}\left(x=\frac{1}{2}\right)$ increases, for example, by the inclusion of $V_{4} \neq 0$.

\section{MONTE CARLO SIMULATIONS}

The Monte Carlo technique has the large advantage that it does include fluctuation effects very accurately, although there are also a number of drawbacks. One problem is that the results are numerical, not providing insight into the dependence, in functional form, of the results on the model 


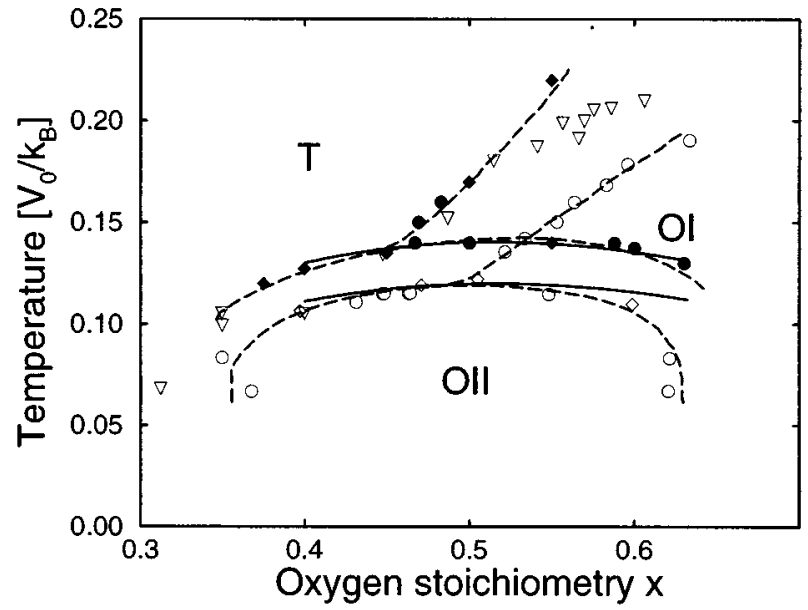

FIG. 4. Comparison of the phase diagrams in the $x$ - $T$ plane obtained by the 2D and the extended 3D ASYNNNI model by Monte Carlo simulation. The results of the fluctuation-corrected mean-field theory are also shown as solid curves. Open circles and diamonds represent Monte Carlo data obtained by Glauber and Kawasaki dynamics, respectively, on the 2D system with interaction parameters $V_{1}=-V_{0}, V_{2}=0.36 V_{0}, V_{3}=-0.12 V_{0}$, and $V_{4}=0$. Solid symbols have the same meaning for the 3D system with $V_{1}, V_{2}$, and $V_{3}$ as before, and $V_{4}=0.02 V_{0}$. The triangles are the experimental data points from Ref. 20. The dashed curves connecting the Monte Carlo data divide the phase diagram into three phases: the disordered tetragonal phase $(T)$, and the two orthorhombic phases ortho-I and ortho-II.

parameters as, e.g., mean-field theory does. Another important problem concerns finite-size effects. The theory of finite-size scaling in particular for thermally driven phase transitions provides a mean for overcoming this problem by extrapolating quantities such as $T_{c}$ obtained from a series of small system sizes. For quantities like the structure factor $S_{\text {dif }}(\mathbf{q})$, the resolution in $q$ space is limited by the system size, ${ }^{52}$ so that finite-size scaling cannot be applied. Therefore in order to resolve details in the line shape, large system sizes with good statistics are needed. This in turn is extremely time consuming (running several weeks on a workstation is not uncommon). Vectorizing the procedure is not easy, because the Monte Carlo algorithm is an inherently sequential process, where the basic computational step consists of randomly selecting a lattice site and updating it according to some transition probability. Nevertheless, in recent years several vectorized and parallel updating algorithms have been devised for speeding up the convergence. One of the problems arising from the use of parallel updating methods is the necessary redefinition of the time variable. However, as far as equilibrium properties are concerned, the sequential and the parallel updating methods lead to the same results. In the present study we have developed a parallel updating algorithm for the 3D extension of the ASYNNNI model, which naturally maps onto the underlying architecture of the Connection Machine CM2.

\section{A. Phase diagram}

We have calculated the phase diagram by Monte Carlo simulation of the Hamiltonian Eq. (9) [the interaction param- eters are given in Eq. (10)], both for the 2D system (where $\left.V_{4}=0\right)$ and for the 3D system. The resulting $x-T$ phase diagram is shown in Fig. 4. The location of the phase boundary was determined by calculating the position of the peak in the fluctuations of the order parameters $\theta_{\mathrm{I}}$ and $\theta_{\mathrm{II}}$ for the two kinds of LRO; see Ref. 23. The Monte Carlo data have been obtained both by Glauber dynamics (circles) and Kawasaki dynamics (diamonds). In the case of Glauber dynamics, $x$ is only indirectly determined from the $x-\mu$ relation. For the $2 \mathrm{D}$ system (open symbols), the system size was $60 \times 60$, while for the $3 \mathrm{D}$ system (solid symbols), the system size was $64 \times 64 \times 8$. To check the finite-size dependence of the results in $2 \mathrm{D}$, larger system sizes $100 \times 100$ and $200 \times 200$ were used at $x=0.4$ and $x=0.5$ for a complete temperature scan across the phase boundary. The differences in $T_{c}$ were small and within the computational uncertainty. For additional computational details about the 2D calculation; see Ref. 23.

The phase diagram contains the high-temperature oxygendisordered tetragonal phase $(T)$ and the two low-temperature oxygen-ordered orthorhombic phases, the ortho-I phase (OI) and ortho-II phase (OII) as indicated in the figure. The dashed lines represent phase boundaries both for the 2D and the 3D systems and are all consistent with being of second order as expected from finite-size scaling analysis of the order parameter discussed by Aukrust et al. ${ }^{39}$ The triangles are the experimental points from Andersen et al. ${ }^{20}$ which have been plotted by using an absolute temperature scale factor of $V_{0} / k_{B}=4490 \mathrm{~K}$. This value has been obtained by fitting the calculated value of $T_{c}(x=0.4)=0.127 V_{0} / k_{B}$ in $3 \mathrm{D}$ to the experimental value $T_{c}=570 \mathrm{~K}$. For this reason the experimental data points fall on the $3 \mathrm{D}$ phase boundary. There is an overall agreement between the experimental data and the 3D ASYNNNI model, although at high temperatures the experimental values falls systematically lower. Additional physics, e.g., inclusion of electronic degrees of freedom ${ }^{53}$ or strain fields, may be needed to account for this deviation. The results of the fluctuation corrected mean-field theory, Eq. (25), are also shown in Fig. 4 as solid lines. It is remarkable that the transition temperature is in close agreement with the Monte Carlo data over a wide range of oxygen stoichiometries. It should also be noted that the theory correctly predicts that the maximum of $T_{c}(x)$ for the ortho-I to ortho-II transition is shifted towards $x>\frac{1}{2}$, and that the shift is larger for the 3D system than for the 2D system because $\stackrel{\circ}{\chi}_{d}$ increases with temperature [cf. Eq. (18)].

\section{B. Structure factor calculations}

The calculation of the structure factors was performed on the CM2 Connection Machine with 65536 single-bit processors. The program was implemented in Connection Machine Fortran (CMF) with calls to the Connection Machine Scientific Software Library (CMSSL). To update the lattice we have used the Metropolis algorithm employing the standard method of dividing the lattice into noninteracting sublattices. Since only one of the chosen sublattices can be updated at a time (without violating detailed balance), each one has been spread across all the available processors of the CM2. Before each calculation of the structure factor, the different sublattices are therefore reassembled into the full lattice. By aligning the different sublattices and the full lattice properly, and 

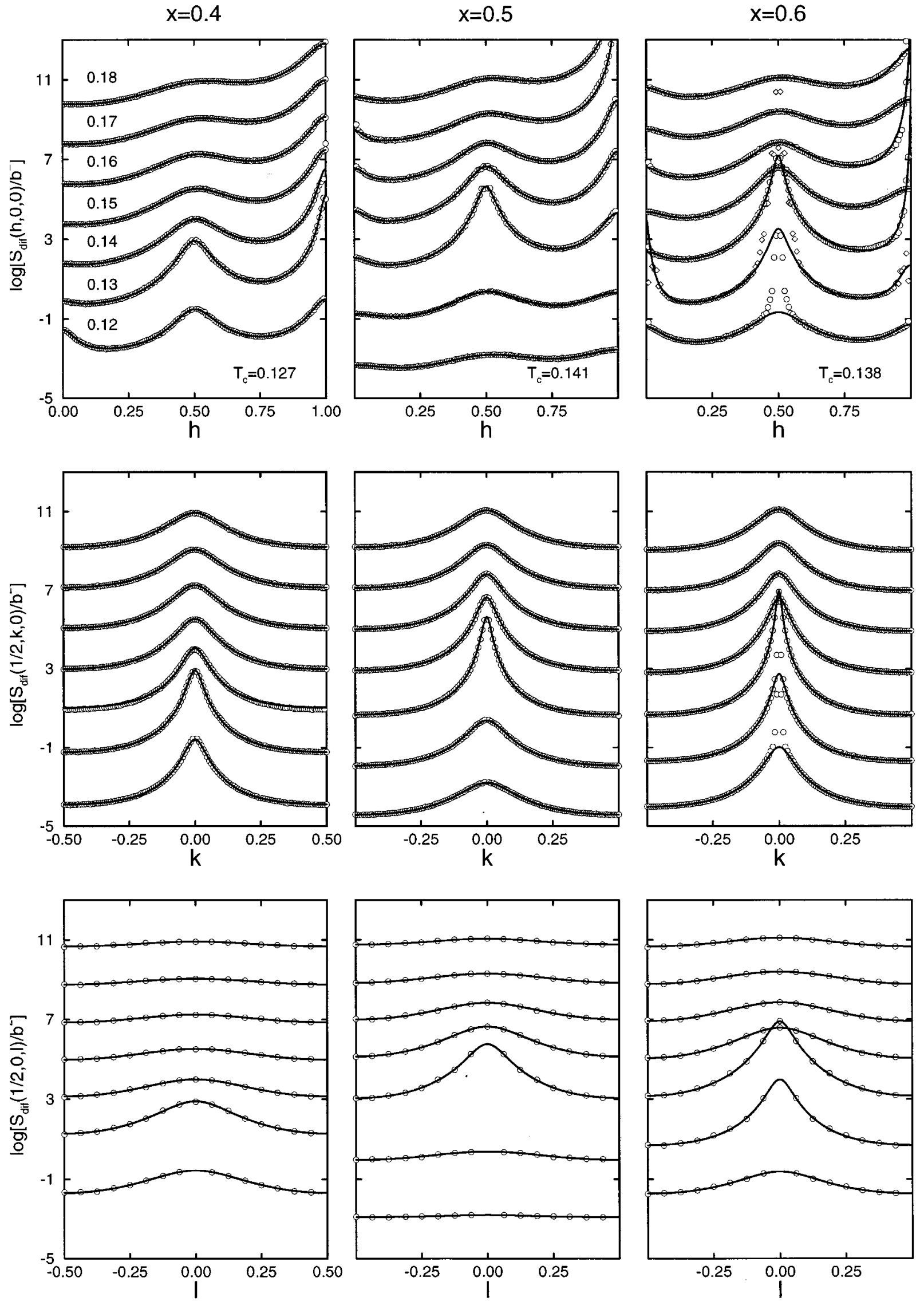

FIG. 5. The line shapes of the $\left(\frac{1}{2}, 0,0\right)$ superstructure reflection $S_{\mathrm{dif}}(q)$ for scans along the main axes in reciprocal space, $(h, k, l)$, are shown on a natural-logarithmic scale for $x=0.4, x=0.5$, and $x=0.6$ for $T=0.12 V_{0} / k_{B}(450 \mathrm{~K})$ to $T=0.18 V_{0} / k_{B}(800 \mathrm{~K})$. The circles are the actual Monte Carlo data points for a 3D system $(256 \times 256 \times 16)$ with interaction parameters $V_{1}=-V_{0}, V_{2}=0.36 V_{0}, V_{3}=-0.12 V_{0}$, and $V_{4}=0.02 V_{0}$, while the solid curves represent the best fits to Eq. (17) plus a diffuse peak centered around $(1,0,0)$ for the ortho-I phase and a diffuse peak at $(0,0,0)$ representing large-scale particle fluctuations. Deviations from the fitted line shapes indicate nonequilibrium domain formations. This is in particular evident at $x=0.6$ due to the increased relaxation time. 
by using the communication commands from the CMSSL, this reassembling process can be carried out without making necessary any data transfer between processors. In the computer simulation the structure factor $S_{\text {dif }}(\mathbf{q})$ has been calculated from Eq. (7) with $\delta \sigma(\mathbf{r})$ replaced by $\sigma(\mathbf{r})$ using a standard fast Fourier transform. This includes the Bragg points in addition to $S_{\text {dif }}(\mathbf{q})$.

For the results of the structure factors reported on below, we have used a system size of $256 \times 256 \times 16$. In the simulations we have used $10^{4}$ Monte Carlo steps per site (MCSS) for equilibration, followed by $5 \times 10^{4}$ MCSS with averaging performed every 10 MCSS. Using 16384 processors, we obtained a peak performance of $8.7 \times 10^{6} \mathrm{MCSS} / \mathrm{sec}$. By application of the symmetry relations pertinent to the oxygen lattice of $\mathrm{YBa}_{2} \mathrm{Cu}_{3} \mathrm{O}_{6+x}$ (space group $P 4 / \mathrm{mmm}$ ), we have reduced the computational efforts of calculating the structure factors to an irreducible zone of reciprocal space which is $1 / 16$ of the Brillouin zone. In addition the symmetry properties of the oxygen lattice were utilized which means that an additional factor of 2 was gained in the statistics by the averaging, giving a total of $1.6 \times 10^{5}$ averages. In Fig. 5 are shown the scans $S_{\text {dif }}(h, 0,0), S_{\text {dif }}\left(\frac{1}{2}, k, 0\right)$, and $S_{\text {dif }}\left(\frac{1}{2}, 0, l\right)$ for three different oxygen stoichiometries $x=0.4, x=0.5$, and $x=0.6$. The scans are presented on a natural-logarithmic scale, and they were performed through the $q$ vector $\mathbf{Q}_{\mathrm{II}}$ $=\left(\frac{1}{2}, 0,0\right)$ corresponding to the characteristic peak for the phase transition of the ortho-II phase. The scans are shown for seven different temperatures ranging from $T=0.12 V_{0} / k_{B}(450 \mathrm{~K})$ to $T=0.18 V_{0} / k_{B}(800 \mathrm{~K})$. The transition temperatures are concentration dependent (cf. Fig. 4), and are given for the three different stoichiometries in the upper panels of Fig. 5. The vertical scale gives the absolute intensities and applies directly to the scans at $T$ $=0.12 V_{0} / k_{B}$. For presentation purposes the remaining scans at $T_{m}=(0.12+0.01 m) V_{0} / k_{B}, \quad m=1, \ldots, 6$, have been shifted vertically upwards by $2 \mathrm{~m}$. The circles are the actual Monte Carlo data points, while the solid curves represent the best fit to the same theoretical expression, Eq. (17), with the simplification that $\chi_{14}\left(\dot{\chi}_{a}, q\right)$ in the denominator is substituted by $\chi_{a} \equiv \chi_{14}\left(\dot{\chi}_{a}, \mathbf{Q}_{\text {II }}\right)$. In order to fit the Monte Carlo data, it is necessary in addition to Eq. (17) to include two diffuse peaks centered around $(0,0,0)$ and $(1,0,0)$, respectively. The $(1,0,0)$ contribution arises partly from SRO ortho-I fluctuations; this contribution could have been incorporated more systematically by including the intricate and cumbersome coupling scheme for three subsystems, as discussed in the Appendix. The $(0,0,0)$ contribution arises from large-scale concentration variations, which have not been accounted for by our mean-field theory. The widths and intensity of these two 3D Lorentzians are adjustable parameters in the fit to the Monte Carlo data. Further, because of the intrinsic limitations of mean-field theory, which prevents a reliable estimate of the $(x, T)$ relations in the phase diagram, we have chosen $\dot{\chi}_{a}$ and $\dot{\chi}_{d}$, and the temperature $T$ in Eq. (17) as free parameters. The very high resolution obtained in the computer simulation allows for an accurate analysis of the line shape, especially in the tails. It follows from Fig. 5 that the theoretical line shape gives an almost perfect fit to the Monte Carlo data for all concentrations and temperatures. In fact this remarkable agreement is not restricted to the scans presented, but includes the entire reciprocal space. This is the reason why the fluctuation-corrected mean-field theory predicts the transition temperatures so accurately at $x \sim \frac{1}{2}$. The reason for the deviations for $x$ very different from $\frac{1}{2}$ is discussed below. The result for the scans $S_{\text {dif }}\left(\frac{1}{2}, k, 0\right)$ and $S_{\text {dif }}\left(\frac{1}{2}, 0, l\right)$ are shown in the two lower panels of Fig. 5. For these scans, it can be shown that the theoretical line shape obtained from Eq. (17) is exactly equal to an infinite sum of Lorentzian line shapes located at $\left(\frac{1}{2}, 2 n+1,0\right)$ and $\left(\frac{1}{2}, 0,2 n+1\right)$ ( $n$ being an integer), respectively. This result is valid within the mean-field theory both above and below $T_{c}$. For $T>T_{c}$ this agrees with the analytical results for both the $1 \mathrm{D}$ and the 2D Ising models. ${ }^{55}$ Let us briefly discuss the systematic behavior reported in Fig. 5, starting with $S_{\mathrm{dif}}\left(\frac{1}{2}, 0, l\right)$. The width $\Delta l$ provides the inverse interplane correlation length $1 / \xi_{c}$. At the oxygen stoichiometry $x=0.4$, it follows that $\xi_{c}$ increases when $T$ approaches $T_{c}$ both from above and below. For $T$ below $T_{c}$, we enter the ordered ortho-II phase (cf. Fig. 4), and there is a $\delta$-function peak at $\left(\frac{1}{2}, 0,0\right)$ (not shown), which gives rise to LRO of ortho-II. A similar scenario is also seen for $S_{\mathrm{dif}}\left(\frac{1}{2}, 0, l\right)$ at $x=0.5$ and $x=0.6$, although the ortho-II fluctuations are present in a LRO of the ortho-I phase, giving rise to a $\delta$-function peak at $(1,0,0)$. Comparing $\xi_{c}$ for different oxygen stoichiometries, at a constant temperature, we notice that for $T=0.12 V_{0} / k_{B}$, the interplane correlation length $\xi_{c}$ has a minimum at $x=0.5$, while it increases both for $x=0.4$ and $x=0.6$. Moving on to $S_{\text {dif }}\left(\frac{1}{2}, k, 0\right)$, the width $\Delta k$ gives the inverse correlation length $1 / \xi_{b}$ within the basal plane along the copper-oxygen chains. Comparing with $S_{\mathrm{dif}}\left(\frac{1}{2}, 0, l\right)$, a similar behavior both in the temperature as well as in the concentration dependence is observed. Again we find extremely good agreement with the theoretical "cosine" line shape both above and below $T_{c}$. Despite the weak interplane interaction parameter $V_{4}$, the system exhibits striking 3D behavior. This is clearly seen when comparing with the $2 \mathrm{D}$ simulation (with $V_{4}=0$ ) ${ }^{56}$ The deviations from the fitted line shape, in particular at $x=0.6$, are due to nonequilibrium domain formations as will be discussed later. In the upper panels of Fig. 5 are shown the results for the $h$ scans, $S_{\text {dif }}(h, 0,0)$. In this direction the width $\Delta h$ of the $\left(\frac{1}{2}, 0,0\right)$ peak carries information about the correlation length $\xi_{a}$ within the basal plane transverse to the copper-oxygen chains. The $\left(\frac{1}{2}, 0,0\right)$ peak shows a similar temperature and concentration dependence as discussed previously. However, the position of the peak is shifted away from the exact $\left(\frac{1}{2}, 0,0\right)$ position, as a result of the nearest neighbor repulsion $V_{1}$, which will be discussed below. For the $h$ scan at $x=0.4$, LRO of ortho-I is not present, (cf. Fig. 4), and the diffuse $(1,0,0)$ peak arises from higher-order reflections of the ortho-II peak in addition to SRO ortho-I. At $x=0.5$ (except for $T=0.18 V_{0} / k_{B}$ ) and for $x=0.6$ there exists LRO ortho-I, which gives rise to an additional $\delta$-function contribution at $(1,0,0)$. It is interesting to note by comparison between the corresponding $k$ and $l$ scans that the difference between SRO or LRO of ortho-I can only be distinguished from the $h$ scan. The importance of the additional $(0,0,0)$ contribution increases for $x$ very different from $\frac{1}{2}$ and if it is neglected in the sum rule, large deviations can be expected, as observed in Fig. 4 for $x \sim x_{p}$ and $x \sim 1-x_{p}$. The simulated structure factor allows us to follow the behavior of the $(0,0,0)$ peak. For $x=0.4$ and $x=0.6$ this 
peak has an entirely different temperature variation than the critical scattering contributing at $\left(\frac{1}{2}, 0,0\right)$ and $(1,0,0)$, since the origin is of geometrical nature. For stoichiometries deviating from $x=\frac{1}{2}$, the connected regions of either holes $(x<$ $\left.\frac{1}{2}\right)$ or particles $\left(x>\frac{1}{2}\right)$ increase as the temperature is reduced, giving rise to more large scale inhomogeneity in the concentration. Therefore, the intensity of the $(0,0,0)$ peaks increases upon decreasing the temperature. In contrast, the behavior at $x=0.5$ shows that the peaks narrow and decrease in intensity with temperature, since the domain walls containing either holes or particles are disfavored at low temperature.

In Figs. 6(a) and 6(b) are shown the widths within the basal plane of the diffuse $\left(\frac{1}{2}, 0,0\right)$ peak at $x=0.4$ as a function of temperature. The widths $\Delta h$ ( $h$ scan $)$ and $\Delta k$ ( $k$ scan) have been calculated for several different system sizes both in $2 \mathrm{D}$ and in $3 \mathrm{D}$. In addition the system size is indicated
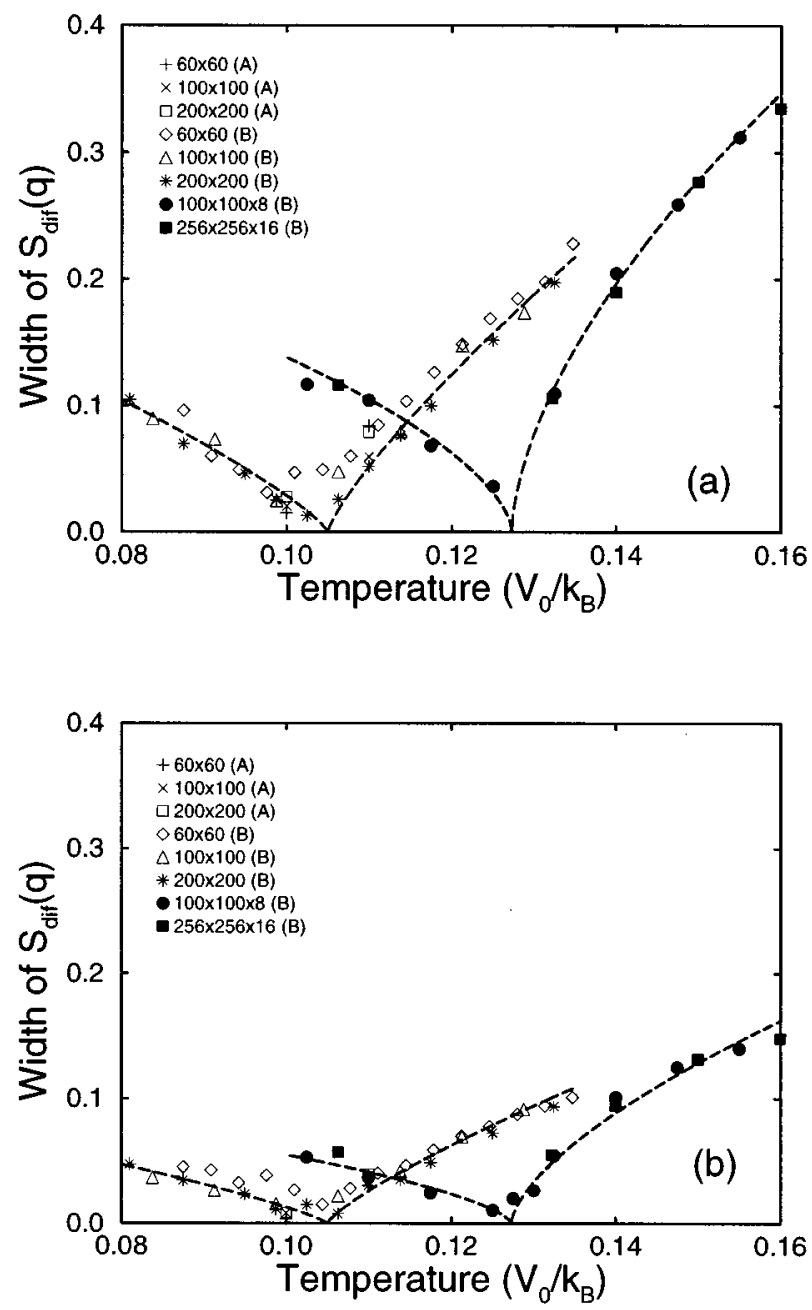

FIG. 6. Comparison between the 2D and the 3D Monte Carlo calculation of the peak width of the diffuse structure factor $S_{\text {dif }}(\mathbf{q})$ at $(1 / 2,0,0)$ as function of temperature for the $h$ scan (a) and the $k$ scan (b), respectively. The oxygen stoichiometry has been fixed to $x=0.4$. The solid symbols represent data points on the $3 \mathrm{D}$ system with interaction parameters $V_{1}=-V_{0}, \quad V_{2}=0.36 V_{0}, \quad V_{3}$ $=-0.12 V_{0}$, and $V_{4}=0.02$, while open symbols are those of the 2D system, using $V_{4}=0.0$. The dashed curves through the data points are the best fits to the scaling relation $\left|T-T_{c}\right|^{\nu}$ with $\nu=0.8$ and $\nu=0.64$ for the $2 \mathrm{D}$ and 3D systems, respectively.
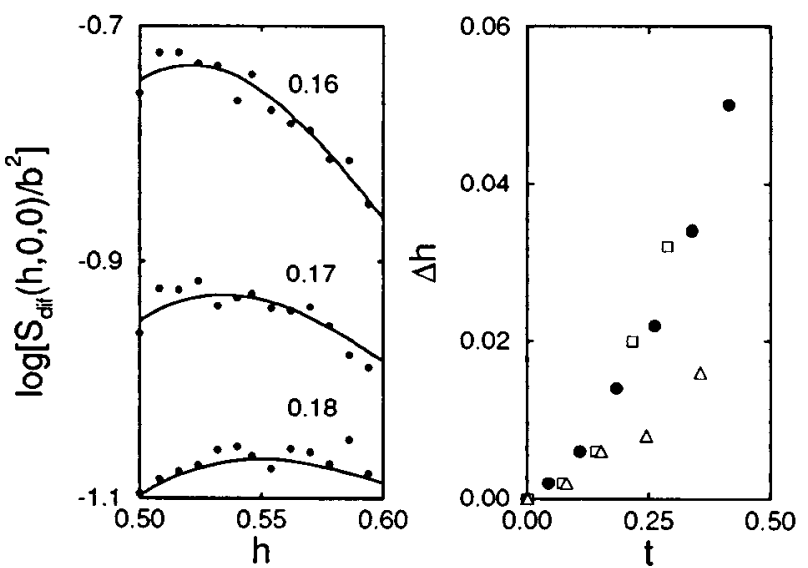

FIG. 7. Right: peak position, $\Delta h+\frac{1}{2}$, of the ortho-II superstructure reflection $S_{\text {dif }}(q)$ for scans along the $h$ axis as function of reduced temperature $t=\left[T-T_{c}(x)\right] / T_{c}(x)$. The system is identical to that in Fig. 5. The symbols denote different oxygen stoichiometry as follows: $x=0.4$ (circles), $x=0.5$ (squares), and $x=0.6$ (triangles). Left: enlargement of the data for $S_{\text {dif }}(h, 0,0)$ including the fits from Fig. 5 at $x=0.4$ for temperatures $T=0.18 V_{0} / k_{B}$ $(t=0.415), \quad T=0.17 V_{0} / k_{B} \quad(t=0.339), \quad$ and $\quad T=0.16 V_{0} / k_{B}$ $(t=0.262)$.

either (A) or (B), referring to the updating dynamics used in the simulations. (A) corresponds to an updating dynamics (Kawasaki exchange dynamics) which is faithful to the local diffusive modes while (B) corresponds to a faster unphysical updating method (Glauber dynamics) allowing oxygenvacancy exchanges over arbitrary distances. However, for equilibrium quantities these two methods yield identical results; see Ref. 23. Although the 2D ASYNNNI model is of Ising type, the universality class of the tetragonal-toorthorhombic ortho-II phase transition has been suggested by Bartelt $e t a l{ }^{57}$ to be that of the $X Y$ model with cubic anisotropy, with an exponent $\nu \approx 0.8$ which depends on the oxygen concentration. The dashed lines in Figs. 6(a) and 6(b) through the 2D data represent a best fit to the data for the largest system using $\nu=0.8$, and using the value for $T_{c}^{2 \mathrm{D}}(x=0.4)=0.1052 V_{0} / k_{B}$; see Ref. 23 . In the 3D case, our numerical data are consistent with the critical exponent $\nu=0.64$ of the 3D Ising model. The dashed curves in Figs. $6(\mathrm{a})$ and $6(\mathrm{~b})$ through the 3D data represent a best fit to the $100 \times 100 \times 8$ data using $\nu=0.64$. It was argued in Sec. II that close to $T_{c}$, where the influence of $\dot{\chi}_{d}$ can be neglected, the in-plane anisotropy $\Delta k / \Delta h=\sqrt{\left|V_{3}\right| /\left|V_{2}\right|}$ is independent of temperature. The Monte Carlo results suggest that the inplane anisotropy $\Delta k / \Delta h$, to a very good approximation is indeed temperature independent. From Figs. 6(a) and 6(b) we obtain the values $\Delta k / \Delta h=0.52$ for the 3D system and $\Delta k / \Delta h=0.50$ for the $2 \mathrm{D}$ system; see Ref. 23 . Hence we find that the inclusion of a weak interplane interaction only marginally affects the anisotropy. We note that the mean-field theory would give a misleadingly larger anisotropy $\Delta k / \Delta h=0.58$. These features are of importance when comparing with the experimental results for the anisotropies.

According to Eq. (18) the line shape of $S_{\text {dif }}(\mathbf{q})$ near a Bragg point $\mathbf{Q}_{\mathrm{II}}$ is modulated by a multiplicative "background" term $1-2 V_{1} \dot{\circ}_{d} q_{x}$, which gives rise to a shift in the 
$\mathrm{x}=0.4$

$\mathrm{T}>\mathrm{T}_{\mathrm{c}}$

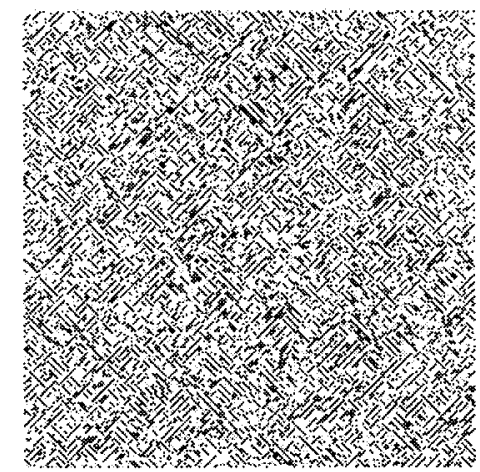

$\mathrm{T}<\mathrm{T}_{\mathrm{c}}$
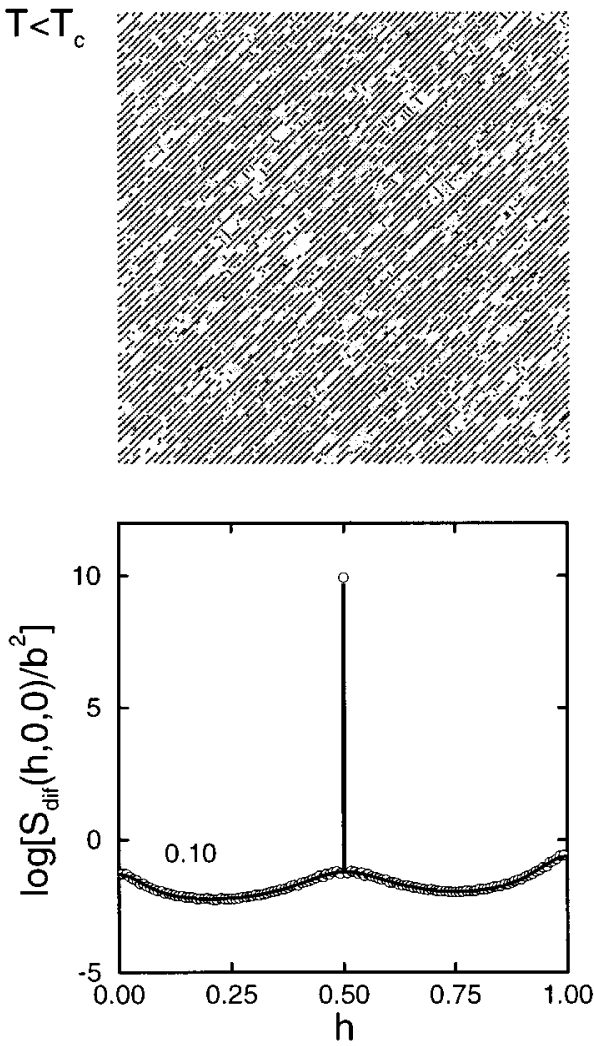

$x=0.5$
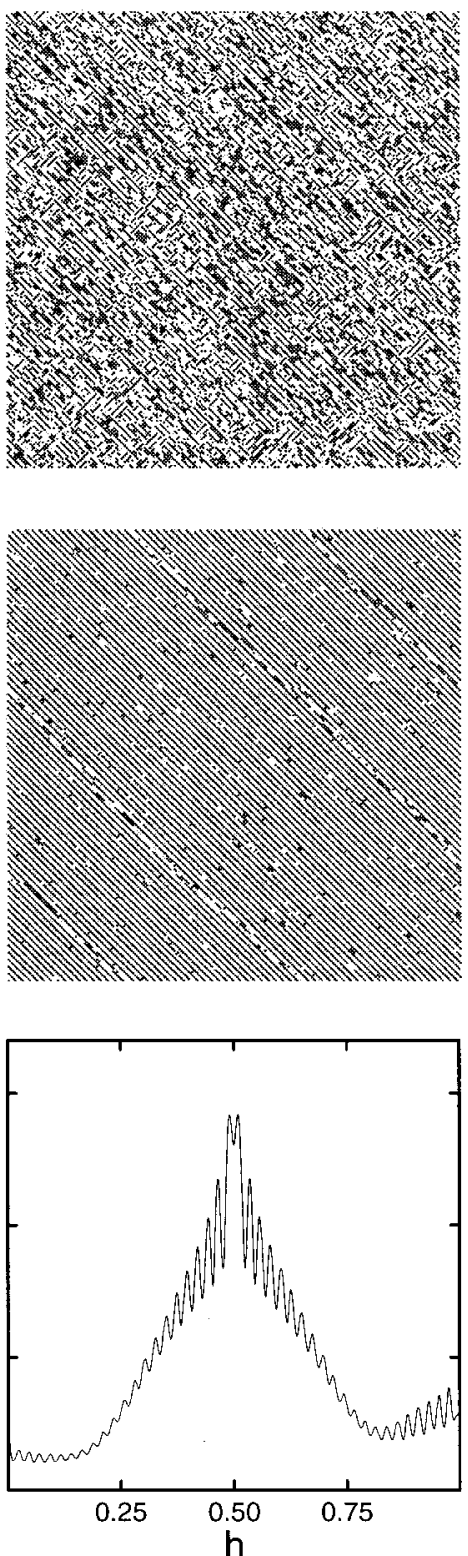

$\mathrm{x}=0.6$
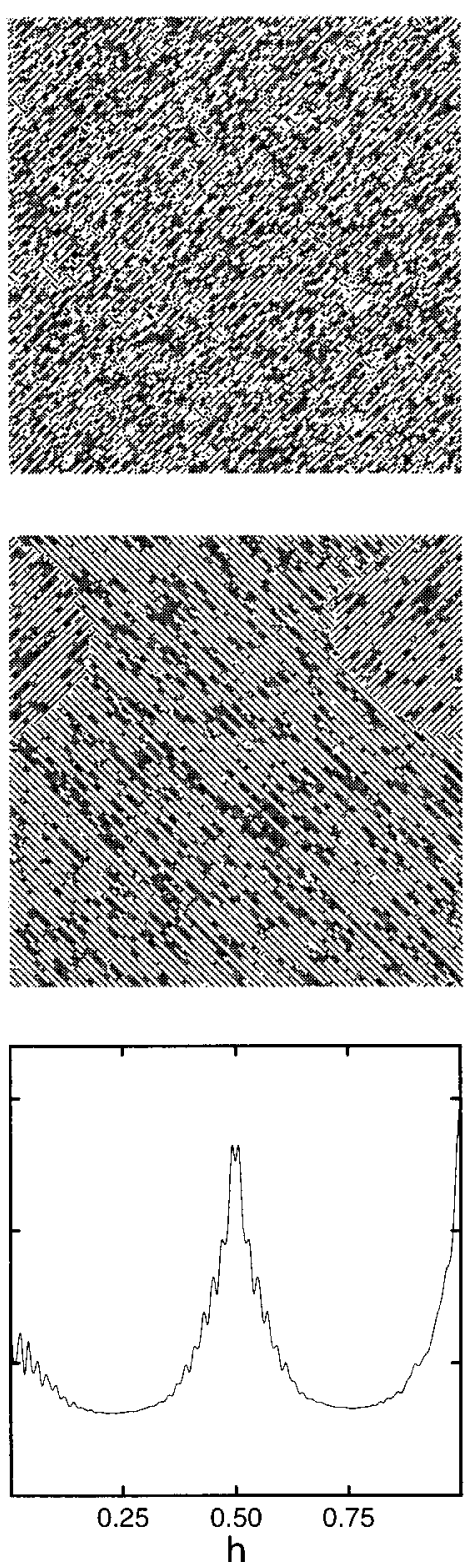

FIG. 8. Real space snapshots of the oxygen configuration in the basal plane for the 3D system $(256 \times 256 \times 16)$ in Fig. 5 , both above and below $T_{c}$, for oxygen stoichiometries $x=0.4, x=0.5$, and $x=0.6$. For the snapshots above $T_{c}$, the reduced temperature was chosen to be the same $t \approx 0.1$, while for those below $T_{c}$, the snapshots represent states at $T=0.10 V_{0} / k_{B}$. The nonequilibrium behavior is most clearly seen for concentrations $x=0.5$ and $x=0.6$. In the last row is shown the low-temperature scans $S_{\text {dif }}(h, 0,0)$ analogous to those presented in Fig. 5. For $x=0.4$ the thick vertical line represent the Bragg contribution. See detailed description in text.

ortho-II superstructure reflection by $\Delta Q_{x}$. In Sec. II it was shown that to first order in $\dot{\chi}_{d}$ the shift is $\Delta Q_{x}=\stackrel{\circ}{\chi}_{d}\left|V_{1}\right| /\left(2\left|V_{3}\right| \chi_{a}\right)$ which depends exponentially on temperature. In Fig. 7 (right) is shown the shift $\Delta Q_{x}$ in the peak position as function of reduced temperature $t=\left(T-T_{c}\right) / T_{c}$, for different oxygen stoichiometries. It is evident that $\Delta Q_{x}$ becomes zero at $t=0$. This is in agreement with the mean-field expression, since at $T_{c}, \chi_{a}$ diverges so that $\Delta Q_{x}$ is identically zero. The predicted exponential dependence is found for $t>0$. In Fig. 7 (left) is shown an enlargement of the profiles for $x=0.4$ at $T=0.16 V_{0} / k_{B}$, $T=0.17 V_{0} / k_{B}$, and $T=0.18 V_{0} / k_{B}$, from which it can be seen that the theoretical line shape agrees with the Monte Carlo data and that the peak position is shifted.

In Fig. 8 is shown real space snapshots of the oxygen configuration in the basal plane for the system in Fig. 5, both above and below $T_{c}$, for oxygen stoichiometries $x=0.4$, $x=0.5$, and $x=0.6$. For the snapshots above $T_{c}$, the reduced temperature was chosen to be the same $t \approx 0.1$, while for those below $T_{c}$, the snapshots represent quenched states at $T=0.10 V_{0} / k_{B}$ for the two largest concentrations. Above $T_{c}(x=0.4)$ only SRO of ortho-I and ortho-II is present, and one can identify domains both along the $a$ and the $b$ axes. Below $T_{c}(x=0.4)$ the SRO ortho-II is replaced by LRO, 
while ortho-I remains as SRO. It is clear from the snapshots that the concentration fluctuations increase upon decreasing the temperature. Below $T_{c}(x=0.4)$ either $a$ or $b$ type domains are selected. The reason is simply that when the correlation length along the chain direction becomes of the order of the system size, percolating domains are formed, and only if these have the same chain direction, can they coexist. In the last row are shown the profiles of the $S_{\text {dif }}(h, 0,0)$ scan, analogous to those presented in Fig. 5. At $x=0.4$ the Bragg contribution is shown as a thick vertical line; this was omitted in Fig 5. Consider the snapshots at $x=0.5$ and $x=0.6$ above $T_{c}$. They both show presence of LRO ortho-I (cf. Fig. 4) and SRO ortho-II. There is clearly a difference in the ortho-II fluctuations compared to those at $x=0.4$. Since already a percolating domain is formed of either $a$ or $b$ type, this disfavors fluctuations of the other type. For $x=0.5$ and $x=0.6$ below $T_{c}$, the system becomes trapped in a nonequilibrium state, where one can identify two percolating domains with the same oxygen chain direction, a phase and an antiphase domain, respectively. Along the $\mathbf{q}=(h, 0,0)$ direction (transverse to the chains), the real space structure can therefore be represented by $\mathcal{F}(r)=A(r) D_{\text {II }}(r)$ $+(1-A(r)) D_{\mathrm{II}}^{\text {anti }}(r)$, where $A(r)$ is approximately a box function with a width $l_{0} a$, which is unity in the ortho-II phase and zero otherwise, while $D_{\mathrm{II}}(r)$ and $D_{\mathrm{II}}^{\text {anti }}(r)$ represent perfect structures of ortho-II and its antiphase. This case was discussed by Fiig et al. ${ }^{54}$ The Fourier transforms of the phase and the antiphase are the same and the total $S_{\text {dif }}^{\text {tot }}(\mathbf{q})=b^{2}|\mathcal{F}(\mathbf{q})|^{2}=2[1-\cos (q a)] S_{\text {dif }}(q)$ is modulated with twice the period of the ortho-II $S_{\text {dif }}(q)$. However, diffuse scattering for a domain of size $l_{0}$ and a lattice constant $a$ is given by $S_{\text {dif }}(q) \propto \sin ^{2}\left(q l_{0} a / 2\right) / \sin ^{2}(q a / 2)$, where $q$ is measured from the ortho-II peaks. Since $l_{0} a$ is of the order of half the system size, this explains the fast oscillations. The actual phase-antiphase domain wall is rough. Accordingly, $A(r)$ must be represented by a narrow distribution of box sizes around the mean $l_{0}$, representing the different domain widths. This gives rise to a nonvanishing envelope function for $S_{\text {dif }}(h, 0,0)$. The width of this function is related to the domain wall roughness. It is not related to the domain width $l_{0}$. In the simulations, the slab domain is stabilized because of finite-size effects and the periodic boundary conditions. ${ }^{58}$ We have tested that the observed domains, both for $x=0.5$ and $x=0.6$, form plates which penetrate the entire system perpendicular to the plane shown. It is counterintuitive that a small interplane coupling can cause such a drastic effect, and it demonstrates the value of performing simulations on realistic system sizes. Similar platelike domains are likely to form in the real $\mathrm{YBa}_{2} \mathrm{Cu}_{3} \mathrm{O}_{6+x}$ material. In experiments one would average over several platelet thicknesses. This averages away the rapid oscillations, except the very first. The resulting diffuse scattering contains information about the roughness of the platelets, but not their widths. Notice further that at $x=0.5$ there is a suppression of the $(0,0,0)$ peak compared to that of $x=0.6$, showing that the concentration variations are largest away from perfect stoichiometry. This is also clearly seen from the snapshots in Fig. 8. At $x=0.6$ the situation is the same as for $x=0.5$. There are also two antiphase domains, although it is difficult to see directly ${ }^{59}$ because in this case the domain walls are more rough. One of these domains is locked by a domain from a perpendicular subsystem (which is easily seen) with copper-oxygen chains along the transverse direction. The corresponding structure factor resembles that of $x=0.5$ although the oscillations are smaller and the peak more narrow due to larger roughness of the walls.

\section{SUMMARY AND CONCLUSION}

We have developed a general mean-field theory for a class of lattice gas Hamiltonians and applied it to the ASYNNNI model. We have shown that the particular nature of this model, having two essentially decoupled subsystems, gives rise to intricate features in the susceptibility $\chi(\mathbf{q})$, basically of geometrical origin, which are very well accounted for by the mean-field theory. The predicted line shape agrees essentially perfectly with the MC data in the complete reciprocal space, above and below the transition temperature $T_{c}$. It further agrees with recent, accurate experimental data above $T_{c}{ }^{8}$ The deviation from the Lorentzian line shapes arising from the sum over all contributions giving the "cosine" form is confirmed by accurate fits to the simulated data on a logarithmic scale. A predicted shift in the peak position of the $(\sim 0.5,0,0)$ peak is demonstrated. The theory is used in conjunction with a sum-rule argument, similar to the spherical approximation used for continuous models, to predict a fluctuation-corrected transition temperature $T_{c}(x)$, which depends both on the dimensionality $D$ and the stoichiometry $x$. This leads to an analytic theory for $T_{c}(x)$ at $x \sim 0.5$ in good agreement with the Monte Carlo results. This allows a direct insight into the parameter dependence of $T_{c}(x)$ without having to resort to time-consuming Monte Carlo calculations. We have performed a series of extensive Monte Carlo calculations. The phase diagram has been determined both in 2D and in 3D by including a small interaction term between adjacent $\mathrm{CuO}_{x}$ planes in $\mathrm{YBa}_{2} \mathrm{Cu}_{3} \mathrm{O}_{6+x}$. A dramatic increase of the transition temperatures $T_{c}(x)$ for all $x$ is found when the small interaction in the third dimension is included. This is of importance when comparisons between first-principles parameters and the experimental data are made. Actually, for the 3D simulations the parameters used to fit the experimental phase transition line separating the tetragonal and orthorhombic phases are in very close agreement with the first-principle in-plane parameters determined by Sterne and Wille. ${ }^{60}$ In contrast to the 2D ASYNNNI model, the 3D extension accounts for an intermediate-temperature range with ortho-I structure before the onset of the ortho-II phase for $x=0.5$, but the suppression is not as pronounced as in the experiments. ${ }^{8}$

Also the line shape changes radically to a Lorentzian shape also below $T_{c}$ in contrast to the $2 \mathrm{D}$ results. ${ }^{54}$ The behavior of the correlation length is found to follow that of the 2D and 3D Ising models, with exponents consistent with the Ising universality classes. The anisotropy in the correlation lengths is found to be independent of $D$ and of $x$. This is important, since the anisotropy is related to the model parameters and can be measured directly in scattering experiments. The in-plane anisotropy is in qualitative agreement with the experimentally observed values, whereas the $c$-axis correlations are much larger than observed experimen- 
tally. This shows that the interplane interaction parameter $V_{4}$, suggested by de Fontaine et al. ${ }^{38}$ and used for the present simulations, is far too large. If $V_{4}$ is reduced $\left(V_{4} \approx 0.004 V_{0}\right)$ to obtain agreement with the experimentally observed anisotropies in the diffuse scattering, $T_{c}$ is reduced by $10 \%$ according to the fluctuation-corrected analytical result presented in Sec. III and Fig. 3. However, for such a small value of $V_{4}$ problems arise in establishing equilibrium in the simulations. In contrast to the 3D simulations the peak width anisotropy ratios obtained experimentally for $x=0.5$ are temperature dependent in the measured range above $T_{c}=400 \mathrm{~K}$ and up to $475 \mathrm{~K}$. Below $T_{c}$ they are constant but the line shape changes gradually from a Lorentzian at $T_{c}$ to a Lorentzian squared at room temperature, which behavior is taken as reminiscent of a 3D random field Ising system. ${ }^{8}$

In the simulations, equilibrium is generally obtained in the smaller systems. In order to obtain high resolution in $\chi(\mathbf{q})$ we have also studied very large systems by using a parallel algorithm, developed for the Connection machine CM2. The high accuracy obtained from the parallel simulations allowed us to carefully test the developed theory. However, it was found that it was sometimes difficult in the simulations to reach the equilibrium state (assumed to be a one domain state). The scattering from the multidomain states shows interesting behavior. It is surprisingly found that the weak interplane coupling is able to stabilize large domains in the third dimension. The observed domains are platelike with rather smooth surfaces. A perfect plane plate of thickness $l_{0}$ would give rise to a $\sin ^{2}\left(q l_{0}\right) / q^{2}$ type scattering instead of a Bragg peak. Roughness and variations in plate thickness would smoothen the oscillations, leaving a broad diffuse peak with a width characteristic of the domain walls, but no Bragg peaks. The width provides no information about the domain size, however. It is possible that the experimentally observed broad peaks (with no Bragg peaks) in the ordered phase of $\mathrm{YBa}_{2} \mathrm{Cu}_{3} \mathrm{O}_{6+x}$ should be understood in this way. This would resolve a long-standing question why long range order is not observed experimentally as Bragg superstructure peaks. ${ }^{8}$ In other less anisotropic materials the domain wall planes are more rough and the above-discussed Fresnel-type oscillations should be less pronounced.

\section{ACKNOWLEDGMENTS}

We thank H. F. Poulsen for support on the 3D Monte Carlo code. The work is supported by the Danish Ministry of Energy, the Danish Natural Science Research Council, and the EEC Science and Esprit programs. The simulations were carried at the Supercomputer Computations Research Institute located at the Florida State University. The research institute is partially funded by the U.S. Department of Energy under Contract No. DE-FC05-85ER250000.

\section{APPENDIX: MEAN-FIELD THEORY FOR A MULTISUBLATTICE SYSTEM}

In this appendix we develop a general mean-field matrix formalism for calculating the phase diagram, susceptibilities, and structure factors of a general class of lattice gas Hamiltonians following Refs. 61,62. The mean-field Hamiltonian is obtained by expansion of Eq. (1) to first order in $\delta \sigma^{\alpha}\left(\mathbf{r}_{\alpha}\right)=\sigma^{\alpha}\left(\mathbf{r}_{\alpha}\right)-c_{\alpha}\left(\mathbf{r}_{\alpha}\right)$, where $c_{\alpha}\left(\mathbf{r}_{\alpha}\right)$ is the mean concentration. We obtain by reintroducing $\sigma^{\alpha}\left(\mathbf{r}_{\alpha}\right)$,

$$
\begin{aligned}
\mathcal{H}^{\mathrm{MF}=}= & \frac{1}{2} \sum_{\alpha, \mathbf{r}_{\alpha}} \sum_{\beta, \mathbf{r}_{\beta}} J^{\alpha \beta}\left(\mathbf{r}_{\alpha}-\mathbf{r}_{\beta}\right) c_{\alpha}\left(\mathbf{r}_{\alpha}\right) c_{\beta}\left(\mathbf{r}_{\beta}\right) \\
& -\sum_{\alpha, \mathbf{r}_{\alpha}} H^{\alpha}\left(\mathbf{r}_{\alpha}\right) \sigma^{\alpha}\left(\mathbf{r}_{\alpha}\right)
\end{aligned}
$$

where

$$
H^{\alpha}\left(\mathbf{r}_{\alpha}\right)=\mu^{\alpha}\left(\mathbf{r}_{\alpha}\right)+\sum_{\beta, \mathbf{r}_{\beta}} J^{\alpha \beta}\left(\mathbf{r}_{\alpha}-\mathbf{r}_{\beta}\right) c_{\beta}\left(\mathbf{r}_{\beta}\right) .
$$

In the form Eq. (A1) the mean-field Hamiltonian can be viewed as a constant term plus a Zeemann term that gives rise to splittings of the energy levels. The mean-field free energy is obtained from the partition function ${ }^{63}$

$$
\mathcal{F}^{\mathrm{MF}}=-k_{B} T \ln \left\{\operatorname{Tr} e^{-\mathcal{H}^{\mathrm{MF}} / k_{B} T}\right\},
$$

and any thermal average with respect to $\mathcal{H}^{\mathrm{MF}}$ is obtained by

$$
\langle O\rangle=\frac{\operatorname{Tr} O e^{-\mathcal{H}^{\mathrm{MF} / k_{B} T}}}{\operatorname{Tr} e^{-\mathcal{H}^{\mathrm{MF} / k_{B} T}} .}
$$

Equation (A3) may be written using the concentrations $c_{\alpha}\left(\mathbf{r}_{\alpha}\right)$ in the conventional form as $\mathcal{F}^{\mathrm{MF}}=\left\langle\mathcal{H}^{\mathrm{MF}}\right\rangle-T \mathcal{S}^{\mathrm{MF}}$, where $\left\langle\mathcal{H}^{\mathrm{MF}}\right\rangle$ is given by Eq. (A1) with $\sigma^{\alpha}\left(\mathbf{r}_{\alpha}\right)$ replaced by the average value $c_{\alpha}\left(\mathbf{r}_{\alpha}\right)$ and the entropy is given by

$$
\begin{aligned}
\mathcal{S}^{\mathrm{MF}}= & -k_{B} \sum_{\alpha, \mathbf{r}_{\alpha}}\left\{c_{\alpha}\left(\mathbf{r}_{\alpha}\right) \ln \left[c_{\alpha}\left(\mathbf{r}_{\alpha}\right)\right]\right. \\
& +\left[1-c_{\alpha}\left(\mathbf{r}_{\alpha}\right) \ln \left(1-c_{\alpha}\left(\mathbf{r}_{\alpha}\right)\right]\right\} .
\end{aligned}
$$

This well-known form for the entropy (see, e.g., Ref. 64) is hence generalized to an arbitrary number of sublattices or constituents. By considering $\mathcal{F}^{\mathrm{MF}}$ as a functional in the variables $c_{\alpha}\left(\mathbf{r}_{\alpha}\right)$, the expectation values of $c_{\alpha}\left(\mathbf{r}_{\alpha}\right)$ can also be determined by minimizing $\mathcal{F}^{\mathrm{MF}}$, which amounts to solving the equations $\partial \mathcal{F}^{\mathrm{MF}} / \partial c_{\alpha}\left(\mathbf{r}_{\alpha}\right)=0$. The result of the minimization procedure yields

$$
H^{\alpha}\left(\mathbf{r}_{\alpha}\right)=k_{B} T \ln \left(\frac{c_{\alpha}\left(\mathbf{r}_{\alpha}\right)}{1-c_{\alpha}\left(\mathbf{r}_{\alpha}\right)}\right),
$$

which is equivalent to the formula we would obtain by applying Eq. (A4), with $\left\langle\sigma^{\alpha}\left(\mathbf{r}_{\alpha}\right)\right\rangle=c^{\alpha}\left(\mathbf{r}_{\alpha}\right)$. Formula (A6) constitutes the generalized Bragg-Williams relation for determining $c_{\alpha}\left(\mathbf{r}_{\alpha}\right)$. For a discussion of discontinuous phase transitions it is necessary to solve Eq. (A4) or Eq. (A6) selfconsistently. The nonlocal susceptibility is defined as

$$
\chi^{\alpha \beta}\left(\mathbf{r}_{\alpha}-\mathbf{r}_{\beta}\right) \equiv \frac{\partial c_{\alpha}\left(\mathbf{r}_{\alpha}\right)}{\partial \mu^{\beta}\left(\mathbf{r}_{\beta}\right)}=\frac{\partial c_{\alpha}\left(\mathbf{r}_{\alpha}\right)}{\partial H^{\alpha}\left(\mathbf{r}_{\alpha}\right)} \frac{\partial H^{\alpha}\left(\mathbf{r}_{\alpha}\right)}{\partial \mu^{\beta}\left(\mathbf{r}_{\beta}\right)},
$$

where we have applied the chain rule. The first term in Eq. (A7) is the local susceptibility; it is diagonal and given by 


$$
\begin{aligned}
\stackrel{\circ}{\chi}_{\alpha} & \equiv \frac{\partial c_{\alpha}\left(\mathbf{r}_{\alpha}\right)}{\partial H^{\alpha}\left(\mathbf{r}_{\alpha}\right)}=\left(\left\langle\sigma^{\alpha}\left(\mathbf{r}_{\alpha}\right) \sigma^{\alpha}\left(\mathbf{r}_{\alpha}\right)\right\rangle-\left\langle\sigma^{\alpha}\left(\mathbf{r}_{\alpha}\right)\right\rangle^{2}\right) / k_{B} T \\
& =\frac{1}{k_{B} T} c_{\alpha}\left(1-c_{\alpha}\right)
\end{aligned}
$$

where in the last equality we have assumed translational symmetry, i.e., $\mu^{\alpha}\left(\mathbf{r}_{\alpha}\right) \rightarrow \mu^{\alpha}$. In this case, differentiation using Eq. (A2) and Eq. (A7) yields

$$
\begin{aligned}
\chi^{\alpha \beta}\left(\mathbf{r}_{\alpha}-\mathbf{r}_{\beta}\right)= & \stackrel{\circ}{\chi}\left\{\delta_{\alpha \beta} \delta_{\mathbf{r}_{\alpha}, \mathbf{r}_{\beta}}+\sum_{\gamma, \mathbf{r}_{\gamma}} J^{\alpha \gamma}\left(\mathbf{r}_{\alpha}-\mathbf{r}_{\gamma}\right)\right. \\
& \left.\times \chi^{\gamma \beta}\left(\mathbf{r}_{\gamma}-\mathbf{r}_{\beta}\right)\right\} .
\end{aligned}
$$

To solve this equation we make use of a Fourier transformation, Eq. (2). By invoking the folding theorem we obtain

$$
\chi^{\alpha \beta}(\mathbf{q})=\stackrel{\circ}{\chi}_{\alpha}\left(\delta_{\alpha \beta}+\sum_{\gamma} J^{\alpha \gamma}(\mathbf{q}) \chi^{\gamma \beta}(\mathbf{q})\right),
$$

which is the self-consistency relation relating the interacting and the noninteracting susceptibility. Equation (A10) may be solved for $\boldsymbol{\chi}(\mathbf{q})$ and expressed in matrix notation as

$$
\chi(\mathbf{q})=\left[\stackrel{\circ}{\chi}^{-1}-\mathbf{J}(\mathbf{q})\right]^{-1}
$$

Consider the situation where the system is separated into two subsystems with different local susceptibilities $\dot{\chi}_{a} \neq \dot{\circ}_{b}$; see Eq. (6). Let us introduce the average susceptibility for thesubsystems consisting of $n$ sublattices $\chi_{\text {av }}(\mathbf{q})$ $\equiv 1 / n \Sigma_{\alpha \beta} \chi^{\alpha \beta}(\mathbf{q})$. From Eq. (6) we obtain the two wavevector-dependent average susceptibilities $\chi_{a}(\mathbf{q}), \chi_{b}(\mathbf{q})$. If the subsystems are coupled by an $n \times n$ matrix $\mathbf{V}(\mathbf{q})$, it can be shown from Eq. (A11) that the total susceptibility is given by

$$
\chi_{\mathrm{av}}(\mathbf{q}) \equiv \frac{1}{n_{s}} \sum_{\alpha \beta} \chi^{\alpha \beta}(\mathbf{q})=\frac{\frac{1}{2}\left\{\chi_{a}^{-1}(\mathbf{q})+\chi_{b}^{-1}(\mathbf{q})\right\}+\bar{V}(\mathbf{q})}{\chi_{a}^{-1}(\mathbf{q}) \chi_{b}^{-1}(\mathbf{q})-\bar{V}(\mathbf{q})^{2}}
$$

where $\bar{V}(\mathbf{q})=1 / n \Sigma_{\alpha \beta}\left\{V^{\alpha \beta}(\mathbf{q})+V^{\beta \alpha *}(\mathbf{q})\right\} / 2$ is the sum over the real part of all matrix elements in $\mathbf{V}(\mathbf{q})$ divided by the number of interacting sublattices. This result also holds if $\chi_{a}(\mathbf{q}) \neq \chi_{b}(\mathbf{q})$ for other reasons, for example, because of different interactions within the subsystems. It is straightforward, but lengthy, to derive the corresponding results for three or more different subsystems with $\chi_{a}(\mathbf{q}) \neq \chi_{b}(\mathbf{q})$ $\neq \chi_{c}(\mathbf{q})$.
*Present address: Danish Meat Research Institute, DK-4000 Roskilde, Denmark.

${ }^{1}$ R. Kikuchi, Phys. Rev. 81, 988 (1951).

${ }^{2}$ D. de Fontaine, in Alloy Phase Stability, Vol. 163 of NATO Advanced Study Institute, Series E: Applied Sciences, edited by G. M. Stocks and A. Gonis (Plenum, New York, 1989).

${ }^{3}$ L. T. Wille, Phys. Rev. B 40, 6931 (1989).

${ }^{4}$ H. F. Poulsen et al., Phys. Rev. Lett. 66, 465 (1991).

${ }^{5}$ D. de Fontaine, L. T. Wille, and S. C. Moss, Phys. Rev. B 36, 5709 (1987).

${ }^{6}$ H. F. Poulsen, N. H. Andersen, and B. Lebech, Physica C 173, 387 (1991).

${ }^{7}$ T. Zeiske et al., Nature 353, 542 (1991).

${ }^{8}$ P. Schleger et al., Phys. Rev. Lett. 74, 1446 (1995).

${ }^{9}$ R. M. Fleming et al., Phys. Rev. B 37, 7920 (1988).

${ }^{10}$ G. Van Tendeloo, H. W. Zandberger, and S. Amelinckx, Solid State Commun. 63, 603 (1987).

${ }^{11}$ M. A. Alario-Franco et al., Physica C 156, 455 (1988).

${ }^{12}$ R. Beyers et al., Nature 340, 619 (1989).

${ }^{13}$ G. Van Tendeloo et al., Mat. Charact. 27, 59 (1991).

${ }^{14}$ J. Reyes-Gasga et al., Physica C 159, 831 (1989).

${ }^{15}$ H. F. Poulsen et al., Nature 349, 595 (1991).

${ }^{16}$ R. J. Cava et al., Physica C 165, 419 (1990).

${ }^{17}$ B. W. Veal et al., Phys. Rev. B 42, 4770 (1990).

${ }^{18}$ J. D. Jorgensen et al., Physica C 167, 571 (1990).

${ }^{19} \mathrm{P}$. Schleger et al., in Proceedings of the $M^{2} S-H T S C-I V$ Conference, Grenoble, France (Elsevier, Amsterdam, 1994).

${ }^{20}$ N. H. Andersen, B. Lebech, and H. F. Poulsen, Physica C 172, 31 (1990).

${ }^{21}$ P. Schleger, W. N. Hardy, and B. X. Yang, Physica C 176, 261 (1991).

${ }^{22}$ D. de Fontaine, M. Asta, G. Ceder, and R. McCormack, Europhys. Lett. 19, 229 (1992).

${ }^{23}$ T. Fiig et al., Physica C 217, 34 (1993).
${ }^{24}$ L. T. Wille, A. Berera, and D. de Fontaine, Phys. Rev. Lett. 60, 1065 (1988).

${ }^{25}$ D. K. Hilton, B. M. Gorman, P. A. Rikvold, and M. A. Novotny, Phys. Rev. B 46, 381 (1992).

${ }^{26}$ T. Fiig, Ph.D. thesis, Ris $\varnothing$ National Laboratory, Denmark, 1994.

${ }^{27}$ R. Sonntag, T. Hohlwein, Brückel, and G. Collin, Phys. Rev. Lett. 66, 1497 (1991).

${ }^{28}$ D. de Fontaine, G. Ceder, and M. Asta, Nature 343, 544 (1990).

${ }^{29}$ T. Krekels et al., Physica C 167, 677 (1990).

${ }^{30}$ G. Ceder, M. Asta, and D. de Fontaine, Physica C 177, 106 (1991).

${ }^{31}$ V. E. Zubkus, S. Lapinskas, and E. E. Tornau, Physica C 166, 472 (1990).

${ }^{32}$ P. Schleger et al., Physica C 241, 103 (1995).

${ }^{33}$ M. A. Krivoglaz, Theory of X-Ray and Thermal-Neutron Scattering by Real Crystals (Plenum Press, New York, 1969).

${ }^{34}$ H. Jagodzinski and F. Frey, in International Tabels for Crystallography, edited by U. Shmueli (Kluwer Academic Publishers, Dordrecht, 1993), Vol. B.

${ }^{35} \mathrm{~S}$. W. Lovesey, Theory of Neutron Scattering from Condensed Matter (Oxford University Press, New York, 1984).

${ }^{36}$ J. V. Andersen, Ph.D. thesis, The Technical University of Denmark, 1991.

${ }^{37}$ H. F. Poulsen, Ph.D. thesis, Ris $\varnothing$ National Laboratory, Denmark, 1991.

${ }^{38}$ D. de Fontaine, G. Ceder, and M. Asta, J. Less-Common Met. 164/165, 108 (1990).

${ }^{39}$ T. Aukrust, M. A. Novotny, P. A. Rikvold, and D. P. Landau, Phys. Rev. B 41, 8772 (1990).

${ }^{40}$ For reasons of simple notation, we shall in the mean-field treatment use reduced lattice vectors and normalize the reciprocal lattice vectors accordingly by taking $a=b=c=1$.

${ }^{41}$ P. A. Lindgård, H. E. Viertiö, and O. G. Mouritsen, Phys. Rev. B 38, 6798 (1988). 
${ }^{42}$ P. A. Lindgård, Phys. Rev. B 27, 2980 (1983).

${ }^{43}$ P. V. Hendriksen, S. Linderoth, and P. A. Lindgård, Phys. Rev. B 48, 7259 (1993).

${ }^{44}$ For the low-dimensional Ising models the problem is actually more complicated. For $D=2$ the exact Onsager solution predicts a marginal order with $T_{c}=0.7 T_{c}^{\mathrm{MF}}$. Because of the large reduction in $T_{c}$ the critical line shape is modified to $S_{\text {dif }}(\mathbf{q}) \propto q^{-2+\eta}$ with $\eta=\frac{1}{4}$ (Ref. 55). This influences strongly the integral Eq. (22). However, for $D=3$ where $\eta \sim 0.05$ the application of the so-called "spherical approximation", should be more reliable, as it is not a marginal case of ordering. We use it here to analytically discuss the influence of the introduction of a coupling in the third dimension.

${ }^{45}$ D. C. Mattis, The Theory of magnetism (John. Weatherhill Inc., New York, 1965).

${ }^{46}$ T. Morita and T. Horiguchi, J. Math. Phys. 6, 981 (1971).

${ }^{47}$ Handbook of Mathematical Functions, edited by M. Abramowitz and I. A. Stegun (Dover Publications Inc., New York, 1972).

${ }^{48}$ A. Berera and D. de Fontaine, Phys. Rev. B 39, 6727 (1989).

${ }^{49}$ C. Baillie, R. Gupta, K. Hawick, and G. Pawley, Phys. Rev. B 45, 10438 (1992).

${ }^{50}$ C. P. Burmester and L. T. Wille, Phys. Rev. B 40, 8795 (1989).
${ }^{51}$ A. A. Aligia, Phys. Rev. B 47, 308 (1993).

${ }^{52}$ E. Vives and P. A. Lindgård, Phys. Rev. B 44, 1318 (1991).

${ }^{53}$ G. Uimin, Solid State Commun. 87, 755 (1993).

${ }^{54}$ T. Fiig, N. H. Andersen, J. Berlin, and Lindgård, Phys. Rev. B 51, 12246 (1995).

${ }^{55}$ M. E. Fisher, Rep. Prog. Phys. 30, 615 (1967).

${ }^{56}$ In contrast, the results show an almost perfect fit to the critical 2D Ising model line shape.

${ }^{57}$ N. C. Bartelt, T. L. Einstein, and L. T. Wille, Phys. Rev. B 40, 10759 (1989).

${ }^{58}$ T. Castan and P. A. Lindgård, Phys. Rev. B 43, 956 (1991).

${ }^{59}$ One can easily visualize the antiphase domains by overlaying the figure with a transparency of the figure.

${ }^{60}$ P. A. Sterne and L. T. Wille, Physica C 162-164, 223 (1989).

${ }^{61}$ P. A. Lindgård, in Neutron Diffraction, edited by H. Dachs (Springer-Verlag, Berlin, 1978).

${ }^{62}$ P. A. Lindgård and O. G. Mouritsen, Phys. Rev. B 41, 668 (1990).

${ }^{63}$ L. D. Landau and E. M. Lifschitz, Statistical Physics (Pergamon Press, Oxford, 1980), Pt. 1.

${ }^{64}$ M. Plischke and B. Bergersen, Equilibrium Statistical Physics (Prentice-Hall International Inc., Englewood Cliffs, NJ, 1989). 